\title{
EQUILIBRIO GENERAL EN ECONOMIAS CON EXTERNALIDADES Y CONJUNTOS DE PRODUCCION NO CONVEXOS EN UN ESPACIO DE BIENES DE INFINITAS DIMENSIONES *
}

\author{
Matías N. Fuentes
}

\begin{abstract}
Resumen
En este trabajo consideramos un modelo de equilibrio general con un espacio de bienes de dimensión infinita, con externalidades que condiciona tanto a los conjuntos de consumo y producción como así también a las preferencias. Estos conjuntos están representados por correspondencias, lo mismo que la conducta de los productores que generaliza a la usada en otros trabajos donde o bien no existen externalidades o bien el espacio de bienes es de dimensión finita. El espacio utilizado es el de las funciones medibles esencialmente acotadas $L_{\infty}(M, \mathrm{M}, \mu)$ de modo que nuestros resultados extienden, entre otros, los trabajos de Bonnisseau (1997) y Bonnisseau-Meddeb (1999).

Clasificación JEL: D 51

Palabras clave: equilibrio general - externaliades

no convexidades - bienes infinitos - correspondencias

* El presente trabajo fue distinguido con el Premio Estudios Económicos 2009, correspondiente a la IV Edición del Congreso Nacional de Estudiantes de Posgrado en Economía, CNEPE. Forma parte de la tesis doctoral del autor en el programa de Doctorado en Economía Teórica de la Universidad Autónoma de Madrid. El autor desea agradecer los valiosos comentarios de los profesores Julio H. G. Olivera, Eduardo Rodríguez y Fernando Tohmé. Todos los errores y omisiones son de mi exclusiva responsabilidad. Con posterioridad a este artículo, el autor desarrolló el teorema aquí expuesto pero relajando varios supuestos lo cual ha implicado que la demostración sea diferente a la aquí desarrollada en algunos tramos de la misma, e-mail: matias.fuentes@unsam.edu.ar.
\end{abstract}




\begin{abstract}
In this paper we consider a general equilibrium model with an infinite dimensional commodity space, with externalities affecting to the consumer and producer sets and the preferences relations. All of these sets are represented by correspondences as well as the producers behavior which extends those used in others papers where there is no externalities or the commodity space is finite dimensional. The space actually used is the one of measurable functions essentially bounded $L_{\infty}(M$, $\mathrm{M}, \mu)$ such that our results generalize, between others, the works of Bonnisseau (1997) and Bonnisseau-Meddeb (1999).
\end{abstract}

JEL Classification: D 51

Keywords: general equilibrium - externalities - non convexities infinitely many commodities - set valued mappings

\title{
INTRODUCCION
}

El propósito del presente trabajo es probar la existencia de equilibrio general para una economía con un espacio de bienes de dimensión infinita, con conjuntos de producción no necesariamente convexos y con la presencia de externalidades que afectan tanto a productores como a consumidores. No debe extrañar esta asociación entre externalidades y no convexidades ya que comúnmente las primeras son causas de las últimas. A modo de ejemplo, citamos un caso tradicional en el que la polución inducida por una firma que tiene un efecto negativo en los consumidores y aún en los planes de producción de las otras firmas que utilizan al agua como un insumo. De modo más general, un efecto externo está definido por el hecho de que la producción de cada productor depende de las producciones de los otros productores así como del consumo de las familias. Con relación al efecto de las externalidades en el consumo de un agente, diremos que este depende de las producciones llevadas a cabo por las empresas y de la corriente de consumo de cada individuo.

Para el caso descrito anteriormente pero con espacio de bienes de dimensión finita, Bonnisseau (1997) y Bonnisseau y Médecin (2001) han obtenido importantes resultados. En el primero de ellos el autor trabaja con una correspondencia de tipo general que define el comportamiento de los 
productores. A dicha correspondencia se le asignan una serie de supuestos que permiten arribar a un vector de precios de equilibrio en la economía. Por su parte el trabajo de Bonnisseau y Médecin (2001) particulariza a la correspondencia general de Bonnisseau (1997) al considerar un concepto extendido del cono normal de Clarke ( 1983) en el que se incluyen las externalidades. La ventaja de mencionada particularización es que prescindimos de los supuestos relativos a la correspondencia que define a la conducta de los productores. En ambos trabajos, y como es común en la teoría del equilibrio general, la existencia de equilibrio es deducida a partir de la existencia de un punto fijo. También cada trabajo generaliza a otros en donde si bien se consideraron tecnologías no convexas, no se incorporaron al análisis las externalidades, así Bonnisseau (1997) extiende el estudio hecho por Bonnisseau-Cornet (1988), en tanto que Bonnisseau y Médecin (2001) generalizan a Bonnisseau y Cornet (1990).

Respecto del espacio de dimensión infinita para los bienes, Bonnisseau y Meddeb (1999) han extendido el trabajo de Bewley (1972) al permitir que los conjuntos sean no convexos. También aquí los autores consideran una correspondencia de tipo general que describe la conducta de los productores y han necesitado supuestos adicionales. Posteriormente Bonnisseau (2000) ha definido al "cono normal grande" en reemplazo de aquella correspondencia lo cual si bien redundó en una menor generalización, permitió excluir los supuestos asociados a dicha correspondencia. Ambos trabajos, aunque siguen la aproximación de Bewley de redes de economías finitas, requieren un tratamiento distinto ya que por las características de la economía original no se deduce un equilibrio en cada economía truncada (Bonnisseau y Meddeb, 1999) o bien este equilibrio existe pero a partir de un espacio de dimensión finita lo suficientemente grande (Bonnisseau, 2000).

El propósito de este trabajo es generalizar los trabajos de Bonnisseau (1997) y Bonnisseau y Meddeb (1999) y tener así un equilibrio, como ya se señaló, para una economía en la cual el espacio de bienes es de dimensión infinita, los conjuntos no son convexos y existen externalidades ${ }^{1}$. Esta generalización no es directa ni rutinaria, sino que la extensión de tales teoremas exige nuevos pasos y esquemas de demostración. Asimismo, a fin de ser consecuentes con la literatura, en caso de que el espacio sea de dimensión finita, nuestro modelo ha de derivar en el de Bonnisseau (1997) y si no existen

\footnotetext{
${ }^{1}$ Obviamente, este modelo no solo extiende a Bonnisseau (1997) y a Bonnisseau y Meddeb (1999) sino a todos aquellos a los cuales estos también generalizan como, por ejemplo, Arrow y Debreu (1954) y Bewley (1972) respectivamente.
} 
externalidades en Bonnisseau y Meddeb (1999). Aquí también seguimos la aproximación de Bewley, sin embargo en cada subeconomía de dimensión finita no tendremos necesariamente un equilibrio sino solo a partir de una cierta dimensión (finita) lo suficientemente grande. El hecho de que los conjuntos no sean convexos y que existan externalidades, no permite una aplicación directa de los teoremas de existencia de equilibrio para ciertas dimensiones finitas.

El trabajo está organizado como sigue: En primer lugar describimos las características de la economía; el espacio de bienes, las externalidades, los precios, los conjuntos de consumo y de producción, la conducta de los productores y la definición de equilibrio general. En segundo término introducimos los supuestos en dicha economía que son suficientes para la existencia de equilibrio. Como la estrategia de demostración pasa por truncar las economías, eso lo hacemos en la sección III. Luego exponemos el teorema de existencia de equilibrio (teorema 4.1) y dos lemas (4.1 y 4.2) relacionados con la existencia de equilibrio en la economía auxiliar de dimensión finita. La sección $\mathrm{V}$ consiste en la demostración del teorema 4.1. Finalmente tenemos los apéndices: el apéndice A consiste en la demostración de los lemas 4.1 y 4.2, con lo que se completa la demostración de que a partir de una cierta dimensión finita cada economía auxiliar cumple con todas las condiciones de existencia de equilibrio de Bonnisseau (1997). Luego, en el apéndice B estudiamos los casos particulares a fin de ser consecuentes con la literatura sobre el tema y poner de manifiesto que el teorema 4.1 generaliza a varios otros en lo concerniente a la existencia de equilibrio; en efecto analizamos qué ocurre con nuestro modelo si (i) el espacio de bienes es de dimensión finita, (ii) si no existen externalidades o bien estas son fijas y (iii) si los conjuntos de producción son convexos. En el apéndice $\mathrm{C}$ exponemos algunas definiciones $\mathrm{y}$ resultados matemáticos utilizados en el presente trabajo.

\section{MODELO DE LA ECONOMIA CON EXTERNALIDADES}

Consideramos una economía con un espacio de bienes infinito dimensional y con una cantidad finita de agentes. Representaremos a dicho espacio por un espacio medida $(M, \mathrm{M}, \mu) \sigma$-finito y positivo. De esta manera el flujo de bienes está definido por funciones medibles de valores reales esencialmente acotadas en $(M, \mathrm{M}, \mu)$. Con estas prescripciones tenemos que el espacio de bienes está dado por $\mathrm{L}_{\infty}(M, \mathrm{M}, \mu)$. La conveniencia de considerar este espacio tanto desde el punto de vista económico como matemático pueden 
consultarse en Bewley (1972) y Mas-Colell y Zame (1991). En particular señalemos que el cono positivo de $\mathrm{L}_{\infty}(M, \mathrm{M}, \mu)$ tiene interior no vacío, de manera que los conjuntos de consumo, si este es el espacio de bienes, gozarán de la misma propiedad ${ }^{2}$. Tanto la definición y el análisis del espacio $\mathrm{L}_{\infty}(M, \mathrm{M}$, $\mu$ ) como así también otros tópicos matemáticos utilizados en el presente trabajo están expuestos, sucintamente, en el apéndice C. En adelante escribiremos a $\mathrm{L}_{\infty}(M, \mathrm{M}, \mu)$ simplemente como $L$.

Una externalidad se representa por toda acción de los agentes económicos, relacionados a actividades de consumo o de producción, que afecta o puede afectar el (buen) comportamiento del resto de los agentes por una vía directa, es decir sin mediar necesariamente los precios (véase, por caso Mas-Colell et al, 1995, pp. 352). En ese sentido, en el presente trabajo consideramos como externalidad al vector que define cualquier corriente de consumo de los $m$ consumidores y la producción llevada a cabo por los $n$ productores.

$$
z=\left(\left(x_{i}\right)_{i=1}^{m},\left(y_{j}\right)_{j=1}^{n}\right) \in L^{m+n}
$$

Este vector define, en la terminología de Debreu (1959), un estado de la economía. Pero tiene una connotación inmediata con el concepto de externalidad, pues cada actor se ve afectado por las decisiones del resto. Así tenemos que la externalidad o el ambiente que afecta al $i$-ésimo consumidor viene dado por:

$$
z_{-i}=\left(\left(x_{1}, x_{2}, \ldots, x_{i-1}, x_{i+1}, \ldots, x_{m}\right),\left(y_{j}\right)_{j=1}^{n}\right) \in L^{m+n-1}
$$

Por su parte la externalidad o estado que condiciona el $j$-ésimo productor es

$$
z_{-j}=\left(\left(x_{i}\right)_{i=1}^{m},\left(y_{1}, y_{2}, \ldots, y_{j-1}, y_{j+1}, \ldots, y_{n}\right)\right) \in L^{m+n-1}
$$

\footnotetext{
2 En el caso de que los conjuntos de producción fueren convexos también estos tendrían un interior no vacío. Todo ello redunda en la existencia de un funcional lineal distinto de cero que permite aplicar uno o varios teoremas de separación (Dunford y Schwarz, 1954, pp. 417-18)
} 
Con esto, decimos que las posibilidades de producción de un productor están representadas por una correspondencia ${ }^{3} Y_{j}: L^{m+n-1} \rightarrow 2^{L} \backslash\{\varnothing\} . \quad Y_{j}\left(z_{-j}\right)$ es el conjunto de producción del $j$-ésimo productor cuando los planes de producción de los otros productores son $y_{1}, y_{2}, \ldots, y_{j-1}, y_{j+1}, \ldots, y_{n}$ y los planes de consumo son $x_{1}, x_{2}, \ldots \mathrm{y} x_{m}$.

Del mismo modo las posibilidades de consumo que tiene el $i$-ésimo consumidor la formalizamos con una función de conjunto $X_{i}$ de $L^{m+n-1}$ en $L$. $X_{i}\left(z_{-i}\right)$ es el conjunto de consumo para el ambiente $z_{-i}$. Los gustos de los consumidores se describen por una relación de preferencias completa, reflexiva, transitiva y binaria, ${ }_{i, z_{-i}}$ en el conjunto $X_{i}\left(z_{-i}\right)$. Sea $x_{i} \in X_{i}\left(z_{-i}\right)$, el conjunto de los elementos de $X_{i}\left(z_{-i}\right)$ estrictamente preferidos a $x_{i}$ es

$$
P_{i}^{+}\left(x_{i}\right)=\left\{x_{i}^{\prime} \in X_{i}\left(z_{-i}\right): x_{i}^{\prime} \succ_{i, z_{-i}} x_{i}\right\}
$$

No asumimos que un individuo pueda comparar dos corrientes de consumos $x_{i}$ y $x_{i}{ }^{\prime}$ si pertenecen a estados $z_{-i}$ y $z_{-i}^{\prime}$ respectivamente y ambos son distintos.

Los precios los ubicamos en el conjunto $S=$ $\left\{\pi \in b a_{+}(M, \mathcal{M}, \mu): \pi\left(\chi_{M}\right)=1\right\}^{4}$, donde $\chi_{M}$ es la función de valor constante 1 para toda $m$ en $M$. La riqueza del consumidor $i$ la definimos por medio de la función compuesta

$r_{i} \mathrm{o} f: S \times L^{n} \rightarrow \mathbb{R}$, con $f\left(\pi,\left(y_{j}\right)_{j=1}^{n}\right)=\left(\pi\left(\omega_{i}\right),\left(\pi\left(y_{j}\right)_{j=1}^{n}\right)\right)$. Es decir, se trata de la composición de dos funciones $f: S \times L^{n} \rightarrow \mathbb{R}^{n+1}$ y $r_{i}: \mathbb{R}^{n+1} \rightarrow \mathbb{R}$. La función de riqueza es dicha composición. Esta captura la idea de la participación de los $m$ consumidores en los dividendos de las empresas como así también incorpora la noción de economía de propiedad privada de Debreu (1959).

\footnotetext{
${ }^{3}$ En lo que sigue hablaremos de correspondencias, funciones multívocas y funciones de conjunto como sinónimos.

${ }^{4} b a(M, \mathrm{M}, \mu)$ que es el dual topológico de $\mathrm{L}_{\infty}(M, \mathrm{M}, \mu)=L$ lo escribiremos también $L^{*}$. El conjunto $S$ es una manera estándar de normalización de precios en espacios de dimensión infinita y puede verse, entre otros trabajos, en los de Bewley (1972), Bonnisseau y Meddeb (1999), Bonnisseau (2000) y Noguchi (1998)
} 
Definimos un estado débilmente eficiente ${ }^{5}$ de la economía por medio del siguiente vector de $L^{m+n}$ :

$Z=\left\{z \in L^{m+n}: \forall i x_{i} \in X_{i}\left(z_{-i}\right), \forall j y_{j} \in \partial_{\infty} Y_{j}\left(z_{-j}\right)\right\}$

La conducta del productor viene dada por una correspondencia $\varphi_{j}: Z \mapsto S .^{6}$ Dado un ambiente $z \in Z$ y una producción débilmente eficiente $y_{j} \in \partial_{\infty} Y_{j}\left(z_{-j}\right), \pi \in L_{+}^{*}$ es un nivel de precios aceptable para el productor $j$ en $y_{j}$ si y solo si $\pi \in \varphi_{j}(z)$. Notemos que esta correspondencia deja abierta la posibilidad a que ciertos productores sean precio aceptantes en tanto que otros sean formadores. Esto último es particularmente cierto en empresas monopólicas que presenten rendimientos crecientes a escala. En el caso de que el conjunto de producción sea convexo tendremos, como caso particular, la conducta maximizadora de beneficios $\varphi_{j}(z)=$ $\left\{\pi \in S: \pi\left(y_{j}\right) \geq \pi\left(y^{\prime}\right), \forall y^{\prime} \in Y_{j}\left(z_{-j}\right)\right\}$.

Las correspondencias $X_{i}$ e $Y_{j}$ fueron definidas sobre el espacio $L^{m+n-1}$. Pero puede ocurrir que ciertos planes $x_{i}$ e $y_{j}$ no sean posibles al mismo tiempo dado que esas acciones no son compatibles entre sí. Por este motivo definimos al conjunto de asignaciones compatibles débilmente eficientes como aquellos en donde la oferta agregada más la dotación inicial de bienes de cada consumidor alcanza para cubrir la demanda de todos ellos. Tenemos que dicho conjunto dado una dotación agregada inicial $\Sigma_{i} \omega_{i}=\omega \in L^{+}$es

$$
A(\omega):=\left\{z \in Z: \sum_{i=1}^{m} x_{i} \leq \sum_{j=1}^{n} y_{j}+\omega\right\}
$$

Finalmente los conjuntos de producción de equilibrio $(P E)$ y de producción de equilibrio factible débilmente eficiente (APE) son respectivamente

\footnotetext{
${ }^{5}$ Bajo el supuesto $\mathrm{P}$ que veremos más adelante, el conjunto de producciones débilmente eficientes del productor $j$ para cada externalidad $z_{-j}$, está en la $\Im$-frontera de $Y_{j}\left(z_{-j}\right)$.

${ }^{6}$ La consideración de función multívoca para expresar el comportamiento del productor es un tema desarrollado en Cornet (1988).
} 
$P E=\left\{(\pi, z) \in S \times Z: \pi \in \bigcap_{j=1}^{n} \varphi_{j}(z)\right\}$

$A P E=\left\{(\pi, z) \in S \times A(\omega): \pi \in \bigcap_{j=1}^{n} \varphi_{j}(z)\right\}$

I. 1. Definición de equilibrio

$(\bar{z}, \bar{\pi})=\left(\left(\bar{x}_{i}\right)_{i=1}^{m},\left(\bar{y}_{j}\right)_{j=1}^{n}, \bar{\pi}\right) \in Z \times S$ es un equilibrio de la economía $\varepsilon=$ $\varepsilon=\left(\left(X_{i},{ }_{i, \mathrm{z}_{-i}}, r_{i}\right)_{i=1}^{m},\left(Y_{j}, \varphi_{j}\right)_{j=1}^{n}, \omega\right) \mathrm{si}:$

1. $\forall i, \bar{x}_{i}$ es un elemento maximal en el conjunto $\left\{x_{i} \in X_{i}\left(\bar{z}_{-i}\right): \bar{\pi}\left(x_{i}\right) \leq r_{i}\left(\bar{\pi}\left(\omega_{i}\right),\left(\bar{\pi}\left(\bar{y}_{j}\right)_{j=1}^{n}\right)\right)\right\}$

2. $\bar{\pi} \in \bigcap_{j=1}^{n} \varphi_{j}(\bar{z})$

3. $\sum_{i=1}^{m} \bar{x}_{i}=\sum_{j=1}^{n} \bar{y}_{j}+\omega$

\section{SUPUESTOS DEL MODELO}

En primer lugar consideramos a la topología $\sigma\left(L, L^{*}\right)$ que es la topología más débil sobre $L$ para la cual todos los elementos de $L^{*}$ son continuos. Dicha topología hace que el espacio topológico $\left(L, \sigma\left(L, L^{*}\right)\right)$ sea de Hausdorff (Ash, 1972, pp. 144). En el mismo sentido la topología $\sigma\left(L^{*}, L\right)$ sobre $L^{*}$ es la topología menos fina (también llamada topología débil estrella o topología débil *) respecto a la cual todos los elementos de $L$ son continuos (aquí los elementos de $L$ se interpretan como funciones de valor real sobre $L^{*}$ ) (Zame y Mas-Colell, 1990, pp. 1839). Con esta topología el espacio topológico $\left(L, \sigma\left(L^{*}, L\right)\right)$ es también de Hausdorff. 
Enunciamos los supuestos que van a utilizarse:

Supuesto C: Sobre los consumidores

(i) $\forall i, X_{i}: L^{m+n-1} \rightarrow 2^{L} \backslash\{\varnothing\}$ es una correspondencia $\sigma\left(L^{m+n-1}, L^{*_{m+n-1}}\right)$-cerrada, valorada convexa y conteniendo al 0 .

(ii) $\forall i$ sea $B$ un conjunto no vacío de $L$ tal que $X_{i}\left(z_{-i}\right) \cap B \neq \varnothing$ entonces existe un $\sigma\left(L^{m+n-1}, L^{*_{m+n-1}}\right)$-entorno de $z_{-i}, U\left(z_{-i}\right)$ tal que $X_{i}\left(z^{\prime}\right) \cap B \neq \varnothing$ para todo $z^{\prime} \in U\left(z_{\text {- }}\right.$ i).

(iii) Para toda $z_{-i} \in L^{m+n-1}$ y $x_{i}$ en $X_{i}\left(z_{-i}\right)$, el conjunto $\left\{x_{i}{ }^{\prime} \in X_{i}\left(z_{-i}\right): x_{i} \precsim_{(i}, z_{-i)} x_{i}{ }^{\prime}\right\}$ es convexo. Para todo entorno de $x_{i}, V\left(x_{i}\right)$, en la topología débil, $P_{i}\left(x_{i}\right) \cap V\left(x_{i}\right) \neq$ $\varnothing$ donde $P_{i}\left(x_{i}\right):=\left\{x_{i}^{\prime} \in X_{i}\left(z_{-i}\right): x_{i} \prec_{(i}, z_{-i)} x_{i}{ }^{\prime}\right\}$ es $\sigma\left(L^{m+n-1}, L^{*_{m+n}-1}\right)$-abierto. Este último es el supuesto de no saciedad local (NS).

(iv) Sea la función de conjunto $\Gamma: L^{m+n-1} \rightarrow 2^{L \times L} \backslash\{\varnothing\}$ definida por $\Gamma\left(z_{-i}\right)=\left\{\left(x_{i}\right.\right.$, $\left.\left.x_{i}{ }^{\prime}\right) \in X_{i}\left(z_{-i}\right)^{2}: x_{i} \precsim_{\left(i, z_{-i}\right.} x_{i}{ }^{\prime}\right\}$. Esta correspondencia es $\sigma\left(L^{m+n-1}, L^{* m+n-1}\right)$ cerrada ${ }^{7}$.

(v) La función $r_{i}$ es continua y estrictamente creciente. Además $\Sigma_{i} r_{i}\left(\pi\left(\omega_{i}\right)\right.$, $\left.\left(\pi\left(y_{j}\right)_{j=1}{ }^{n}\right)\right)=\pi(\omega)+\sum_{j} \pi\left(y_{j}\right)$ y si $z \in A(\omega)$ entonces $r_{i}\left(\pi\left(\omega_{i}\right),\left(\pi\left(y_{j}\right)_{j=1}{ }^{n}\right)\right)>0$.

Supuesto P: Sobre los productores

(i) $\forall j, Y_{j}: L^{m+n-1} \rightarrow 2^{L} \backslash\{\varnothing\}$ es una correspondencia $\sigma\left(L^{m+n-1}, L^{* m+n-1}\right)$-cerrada.

(ii) $\forall j$ sea $B$ un conjunto no vacío de $L$ tal que $Y_{j}\left(z_{-j}\right) \cap B \neq \varnothing$ entonces existe un $\sigma\left(L^{m+n-1}, L^{* m+n-1}\right)$-entorno de $z_{-j}, U\left(z_{-j}\right)$ tal que $Y_{j}\left(z^{\prime}\right) \cap B \neq \varnothing$ para todo $z^{\prime} \in U\left(z_{\text {- }}\right.$ j).

(iii) Existen $\underline{t}_{j} \mathrm{y} t_{j}^{\prime} \in \mathbb{R}$ tal que para toda $z_{-j} \in L^{m+n-1} \underline{t}_{j} \chi_{M} \in Y_{j}\left(z_{-j}\right)$ y $t_{j}^{\prime} \chi_{M} \notin Y_{j}\left(z_{-j}\right)$

\footnotetext{
${ }^{7}$ Sea, para cada $z_{-i}$, el conjunto $\left.\Gamma^{+}\left(z_{-i}\right)=\left\{\left(x_{i}, x_{i}{ }^{\prime}\right) \in X_{i}\left(z_{-i}\right)^{2}: x_{i} \succ{ }_{(i}, z_{-i)} x_{i}{ }^{\prime}\right\}\right\} .\left\{\left(z^{*}{ }_{-i}, x_{i}{ }^{*}, x_{i}{ }^{\prime}\right) \in L^{m+n+1}\right.$ $\left.:\left(x_{i}^{*}, x_{i}^{\prime}\right) \in \Gamma^{+}\left(z_{-i}^{*}\right)\right\}$ pertenece a un conjunto $\sigma\left(L^{m+n-1}, L^{* m+n-1}\right)$-abierto de $L^{m+n+1}$.
} 
(iv) $Y_{j}\left(z_{-j}\right)-L_{+}=Y_{j}\left(z_{-j}\right)$ para toda $z_{-j} \in L^{m+n-1}$ (libre disponibilidad).

\section{Supuesto B}

El conjunto $A\left(\omega^{\prime}\right):=\left\{z \in Z: \Sigma_{i} x_{i} \leq \Sigma_{j} y_{j}+\omega^{\prime}\right\}$ donde $\omega^{\prime} \geq \omega$ es acotado en norma.

Notemos que en ausencia de externalidades, los supuestos C(i) y C(iii)-(iv) son prácticamente similares al supuesto $\mathrm{C}$ de Bonnisseau y Meddeb (1999). Para cada ambiente fijo $z_{-i} \in L^{m+n-1}, X_{i}\left(z_{-i}\right)$ es cerrada en la topología débil (Joseph, 1978, pp. 509-510). El supuesto C(iv) es la extensión natural del supuesto de preferencias continuas al caso presente en que trabajamos con correspondencias de consumo en lugar de conjuntos. Por supuesto, ello implica continuidad débil dado un ambiente fijo o ausencia de externalidades ${ }^{8}$. En C(v) se tiene que si la riqueza global es positiva entonces el ingreso de cada individuo es positivo. A menudo también se ha argumentado que este supuesto revela que existe un mecanismo institucional por el cual las pérdidas de las firmas con tecnologías no convexas son compensadas. El supuesto C(ii) merece una consideración aparte: obsérvese que se trata de un supuesto más fuerte que el de hemicontinuidad débil ya que aquel vale para todo subconjunto $B$ tal que $X_{i}\left(z_{-i}\right) \cap B \neq \varnothing$, mientras que en el caso de la hemicontinuidad se exige que $B$ sea abierto. Si tal es el caso de $B$, entonces las condiciones supuestas implican la hemicontinuidad inferior. Más aún este supuesto tiene como caso particular que si $x \in X_{i}\left(z_{-i}\right)$ entonces vale decir que $\{x\} \subset X_{i}\left(z_{-i}\right)$ y por lo tanto existe un $\sigma$-entorno de $z_{-i}, U\left(z_{-i}\right)$, tal que $x \in X_{i}\left(U\left(z_{-i}\right)\right)$. Es decir dada una externalidad que define un conjunto de consumo, si un bien o servicio se encuentra en dicho conjunto se encontrará también en otro conjunto de consumo condicionado por una externalidad "cercana" a aquella. De no haber externalidades o bien si estas son fijas ambos conceptos coincidirían. Así el teorema que aquí desarrollamos generaliza el trabajo, entre otros, de Bewley (1972), Bonnisseau (1997), Bonnisseau y Cornet(1988) y Bonnisseau y Meddeb (1999).

Respecto del supuesto P, acontece lo mismo que lo comentado en el caso de la correspondencia $X_{i}$ en los supuestos C(i)-(iv) ${ }^{9}$, tanto si no existen

\footnotetext{
${ }^{8}$ Pues tendríamos un conjunto convexo en donde la topología débil coincide con la topología Mackey o topología fuerte entre las localmente convexas y de Hausdorff. Para una definición precisa de la topología Mackey véase Nowak, M. (1989), teoremas 2 y 5 . Respecto de la equivalencia de topologías puede consultarse Shcaefer (1971).

${ }^{9}$ Excepto, claro está, en lo relacionado a la valoración convexa.
} 
externalidades como si estas son fijas. En efecto, en cualquiera de estos dos casos tendríamos que $Y_{j}$ es un conjunto débilmente cerrado. La asunción de libre disponibilidad implica que dada una externalidad $z_{-j} \in L^{m+n-1}$, las producciones débilmente eficientes se encuentran en la frontera de $Y_{j}\left(z_{-j}\right)$ para la topología inducida por la norma. El supuesto P(iii) no está contemplado en el trabajo de Bonnisseau y Meddeb (1999) pero guarda relación con Bonnisseau (1997) y ha de usarse cuando trunquemos la economía original en subeconomías de dimensión finita. Notemos que el supuesto P(iii) al afirmar que $t_{j} \chi_{M} \in Y_{j}\left(z_{-j}\right)$ para toda $z_{-j} \in L^{m+n-1}$ es más débil que suponer que $-L_{+} \subset Y_{j}\left(z_{\text {- }}\right.$ $j$ ), ya el nuestro sería un caso particular de este último. También asumir que $t_{j}^{\prime} \chi_{M} \notin Y_{j}\left(z_{-j}\right)$ es menos restrictivo que asumir la imposibilidad de producción libre (Debreu, 1959). Obviamente lo que estos supuestos quieren hacer notar es que no se puede incrementar la producción sin hacer lo propio con los inputs dado un ambiente o externalidad.

El supuesto B aquí es similar a Bonnisseau y Meddeb (1999) con la terminología de Bonnisseau (1997). Por otra parte, el supuesto B asume la acotación dada para toda $\omega^{\prime} \geq \omega$ y no solamente para $\omega$. Ello es así puesto que si supusiéramos solo para $\omega$ Bonnisseau-Cornet (1988) han dado un ejemplo de que el equilibrio puede no existir debido a las posibilidades de pérdida estricta (pp. 146, remark 5.1).

Supuesto BL (pérdida acotada)

Para todo productor $j=1, \ldots, n$; existe un número real $\alpha_{j}$ tal que, para todo $z \in$ $Z$ y para todo $\pi_{j} \in \varphi_{j}(z), \pi_{j}\left(y_{j}\right) \geq \alpha_{j}$.

Supuesto WSA (supuesto de supervivencia débil)

Para todo $(\pi, z, \lambda) \in \mathrm{PE} \times \mathbb{R}_{+}$, si $z \in A\left(\omega+\lambda \chi_{M}\right)$ entonces $\pi\left(\Sigma_{j} y_{j}+\omega+\lambda \chi_{M}\right)>0$

Notemos que si $z \in A\left(\omega+\lambda \chi_{M}\right)$ entonces $\Sigma_{j} y_{j}+\omega+\lambda \chi_{M} \geq 0$ y por lo tanto $\pi\left(\Sigma_{j}\right.$ $\left.y_{j}+\omega+\lambda \chi_{M}\right) \geq 0$ para todo $\pi \in L^{*}{ }^{*}$. Así, lo que el supuesto WSA dice es que el nivel común de precios de la economía dado por la regla de asignación de precios de cada productor permite obtener un nivel de riqueza global que está por encima del mínimo absoluto. Como se sabe, este supuesto es un debilitamiento del supuesto de supervivencia SA donde $\lambda=0$. (Kamiya, 1988, pp. 263). 


\section{SUBECONOMIAS DE DIMENSION FINITA}

\section{III.1. Definiciones y propiedades básicas}

Consideremos una red $F$ de subespacios de dimensión finita de $L$ dirigida por inclusión y conteniendo tanto a $\left(\omega_{i}\right)$ como a $\chi_{M}$. Para cada $F \in F$ tenemos que $F^{*}$ es su dual; además $F$ hereda el producto interior de $L$, y como $F_{+} \cap\left(-F_{+}\right)=\{0\}$ entonces $F_{+}$es un cono propio. Notemos que en la topología inducida $\operatorname{int} F_{+}=F \cap \operatorname{int} L_{+}$que es no vacío ya que $\chi_{M}$ está en $\operatorname{int} L_{+}$. De ahí que para todo $F \in F$ y para todo $n \in \mathbb{N}$ tenemos que $F$ con $\operatorname{dim}(F)=n$ es equivalente al espacio euclídeo $n$ dimensional $\mathbb{R}^{n}$ (Dunford y Schwartz, 1958, pp. 245 $)^{10}$. Con estos preceptos podemos asignar a $F$ una estructura euclídea ${ }^{11}$ tal que $\chi_{M}{ }^{{ }^{F}}=\left\{\xi^{F} \in F: \xi^{F}\left(\chi_{M}\right)=0\right\}, \chi_{M^{\perp}}{ }^{F} \cap F_{+}=\{0\} \mathrm{y}\left\|\chi_{M}{ }^{F}\right\|=1$.

A continuación truncaremos la economía de dimensión infinita en subeconomías finitas. A tales fines consideraremos las siguientes correspondencias

$X_{i}^{F}:(L \cap F)^{m+n-1} \rightarrow 2^{L \cap F \backslash\{\varnothing\} .}$

$Y_{j}^{F}:(L \cap F)^{m+n-1} \rightarrow 2^{L \cap F} \backslash\{\varnothing\}$.

Definidas, respectivamente, por

$X_{i}^{F}\left(z^{F}{ }_{-i}\right)=X_{i}\left(z^{F}{ }_{-i}\right) \cap F$.

$Y_{j}^{F}\left(z^{F}{ }_{-i}\right)=Y_{j}\left(z^{F}{ }_{-i}\right) \cap F$.

Asimismo tenemos que $S \cap F^{*}{ }_{+}=S^{F}=\left\{\pi \in F_{+}^{*}: \pi\left(\chi_{M}\right)=1\right\}, F_{+}=$ $F \cap L_{+}$e $\operatorname{int} F_{+}=F \cap \operatorname{int} L_{+}$. Veamos a continuación algunas cuestiones importantes relacionadas con el funcionamiento de estas economías truncadas o subeconomías. En primer lugar notemos que para todo $F \in \mathcal{F}, X_{i}\left(z^{F}{ }_{-i}\right) \cap F \neq \varnothing$

\footnotetext{
${ }^{10}$ En realidad $F$ es equivalente a $E^{n}$, el espacio lineal de $n$-tuplas ordenadas. Dado que nosotros estamos considerando el campo de escalares reales es que hemos tomado $\mathbb{R}^{n}$.

${ }^{11}$ Aquí la estructura euclidea sobre $F$ no es la misma que la de $\mathbb{R}^{n}$, pero será suficiente con remplazar el vector $e=(1,1, \ldots, 1)$ en Bonnisseau (1997) por $\chi_{M}$.
} 
ya que por C(i) $0 \in X_{i}\left(z^{F}{ }_{-i}\right)$ y como $F$ es un subespacio de $L, 0 \in F$. También para todo $F \in F \quad Y_{j}\left(z^{F}{ }_{-j}\right) \cap F \neq \varnothing$ ya que por el supuesto $\mathrm{P}(\mathrm{ii}) \underline{t}_{j} \chi_{M} \in Y_{j}\left(z^{F}{ }_{-j}\right)$ y como $F$ es un subespacio que contiene a $\chi_{M}, \underline{t}_{j} \chi_{M} \in F$.

En segundo término veamos que cualquiera sea $F \in F \quad \partial\left(Y_{j}\left(z^{F}{ }_{-j}\right) \cap F\right) \subset$ $\partial_{\infty} Y_{j}\left(z^{F_{-j}}\right)$, para ello baste con probar que $\partial\left(Y_{j}\left(z_{-j}^{F}\right) \cap F\right)=\partial_{\infty} Y_{j}\left(z^{F_{-j}}\right) \cap F$. En efecto si $y_{j} \in \partial_{\infty} Y_{j}\left(z^{F}{ }_{-j}\right) \cap F$ es evidente que $y_{j} \in \partial\left(Y_{j}\left(z^{F}{ }_{-j}\right) \cap F\right)$. A la inversa sea $y_{j} \in$ $\partial\left(Y_{j}\left(z^{F}{ }_{-j}\right) \cap F\right)$ entonces $y_{j} \in Y_{j}\left(z^{F}{ }_{-j}\right)$ e $y_{j} \in F$. Por P(iii) existe $t_{j}^{\prime} \in \mathbb{R}$ tal que $t_{j}^{\prime} \chi_{M}$ $\notin Y_{j}\left(z^{F}{ }_{-j}\right)$. Sea $t^{*}>\max \left\{t_{j}^{\prime},\left.\left|y_{j}\right|\right|_{\infty}\right\}$, de ahí tenemos que $t^{*} \chi_{M}>t_{j}^{\prime} \chi_{M}, t^{*} \chi_{M}>y_{j} \mathrm{y}$ por $\mathrm{P}(\mathrm{iv}) t^{*} \chi_{M} \notin Y_{j}\left(z^{F}{ }_{-j}\right)^{12}$. Como $F$ es un subespacio $t^{*} \chi_{M}$ está en $F$, lo mismo que el funcional $\alpha y_{j}+(1-\alpha) t^{*} \chi_{M}$ para todo $\alpha$ en $[0,1]$. Si $\alpha=1$ este funcional está en $Y_{j}\left(z^{F}{ }_{-j}\right)$ y si $\alpha=0$ entonces estará en $L \backslash Y_{j}\left(z^{F}{ }_{-j}\right)$. De ahí que para algún $\alpha$ $\in(0,1] \alpha y_{j}+(1-\alpha) t^{*} \chi_{M} \in \partial_{\infty} Y_{j}\left(z^{F}{ }_{-j}\right) \cap F$. Notemos que el único caso que puede darse es $\alpha=1$. En efecto si $\alpha<1$ entonces tendríamos que $\alpha y_{j}+(1-\alpha) t^{*} \chi_{M} \in$ $\partial_{\infty} Y_{j}\left(z^{F}{ }_{-j}\right) \cap F \subset \partial\left(Y_{j}\left(z_{-j}^{F}\right) \cap F\right)$ por lo que se probó en la primera parte, pero $\alpha y_{j}+$ $(1-\alpha) t^{*} \chi_{M}>y_{j} \mu$-c.t.p. lo cual contradice que $y_{j} \in \partial\left(Y_{j}\left(z^{F}{ }_{-j}\right) \cap F\right)$. Luego $y_{j} \in$ $\partial_{\infty} Y_{j}\left(z_{-j}^{F}\right) \cap F$.

Tercero, notemos que el conjunto que designa los estados truncados de la economía débilmente eficientes está dado por $Z^{F}=\left\{z^{F} \in(L \cap F)^{m+n}: \forall i x_{i}^{F} \in\right.$ $X_{i}\left(z^{F}{ }_{-i}\right) \cap F$ y $\left.\forall j y_{j}^{F} \in \quad \partial\left(Y_{j}\left(z^{F}{ }_{-j}\right) \cap F\right)\right\}$ el cual está contenido en el conjunto $Z$.

En cuarto lugar definimos la correspondencia $\varphi_{j}^{F}: Z^{F} \mapsto 2^{S \cap F^{*}+} \backslash\{\varnothing\}$ expresada como $\varphi_{j}^{F}\left(z^{F}\right)=\varphi_{j}\left(z^{F}\right) \cap F^{*}$. Veamos que esta función multívoca está bien definida ya que si $\pi \in \varphi_{j}\left(z^{F}\right) \subset S$, entonces $\pi: L_{+} \mapsto \mathbb{R}$ y en particular $\pi$ está definida en $F$, o sea $\left.\pi\right|_{F}: L_{+} \cap F \mapsto \mathbb{R}$, por lo que $\left.\pi\right|_{F} \in F^{*}{ }_{+}$. Como $\chi_{M} \in F$, $\left.\pi\right|_{F}\left(\chi_{M}\right)=\pi\left(\chi_{M}\right)$ por lo que $\varphi_{j}\left(z^{F}\right) \cap F^{*}+\subset S^{F}$. En otras palabras $\varphi_{j}^{F}$ es la restricción de todo elemento de $\varphi_{j}$ a $F$ o lo que es lo mismo, para todo elemento de $\varphi_{j}^{F}$ existe una extensión a $L$ que está en el conjunto $\varphi_{j}$.

En quinto término, veamos algunas cuestiones consideradas con la externalidad o ambiente y la inclusión. Consideremos por tanto un espacio de bienes de dimensión $r, F_{r}$. Notemos que $F_{r} \subset F_{r+k}, k \in \mathbb{N}$. En consecuencia $z^{F r}{ }_{-i}$ $\in \prod_{i=1}^{m-1}\left(L \cap F_{r}\right) \times \prod_{j=1}^{n}\left(L \cap F_{r}\right) \subset \prod_{i=1}^{m-1}\left(L \cap F_{r+k}\right) \times \prod_{j=1}^{n}\left(L \cap F_{r+k}\right)$. Ahora bien,

\footnotetext{
${ }^{12}$ Sea $c=t^{*}-t_{j}^{\prime}>0$, de modo que $t^{*} \chi_{M}-c \chi_{M}=t_{j}^{\prime} \chi_{M}$. Si suponemos que $t^{*} \chi_{M} \in Y_{j}\left(z^{F}{ }_{-j}\right)$ entonces por el supuesto de libre disponibilidad también $t^{*} \chi_{M}-c \chi_{M}$, lo cual contradice que $t_{j}^{\prime} \chi_{M} \notin Y_{j}\left(z_{-j}^{F}\right)$.
} 
sean $z^{F r}{ }_{-i}$ y $z^{r F r}{ }_{i}$ en $\prod_{i=1}^{m-1}\left(L \cap F_{r}\right) \times \prod_{j=1}^{n}\left(L \cap F_{r}\right)$; dado que los conjuntos de consumo no necesariamente son los mismos cuando cambia el ambiente, no podemos comparar dos corrientes de consumos que pertenecen a externalidades diferentes, por ello $X_{i}^{F r}\left(z^{F r}{ }_{-i}\right)$ no es necesariamente igual a $X_{i}^{F r}\left(z{ }^{F r}{ }_{-i}\right)$. Como consecuencia tendremos que a diferencia de lo que ocurre en los trabajos donde se sigue la aproximación por economías finitas sin externalidades aquí $X_{i}^{F r}\left(z^{F r}{ }_{-i}\right)$ no tiene por que estar incluido en $X_{i}^{F r+k}\left(z^{F r+k}{ }_{-i}\right)^{13}$. Es decir aunque la familia $F$ esté dirigida por inclusión no sucederá directamente lo mismo con los conjuntos de consumo y producción ya que, formalmente, $z^{F r}{ }_{-i}$ y $z^{F r+k}{ }_{-i}$ son elementos de $\left(L \cap F_{r+k}\right)^{m+n-1}$ y por tanto se pueden considerar como ambientes distintos. Matemáticamente decimos que es dable tener $X_{i}\left(z^{F r}{ }_{-i}\right) \cap F_{r} \subset X_{i}\left(z^{F r}\right.$. $\left.{ }_{i}\right) \cap F_{r+k} \not \subset \quad X_{i}\left(z^{F r+k}{ }_{-i}\right) \cap F_{r+k}{ }^{14}$.

Así, los conjuntos de asignaciones débilmente eficientes y factibles, de equilibrio y de equilibrio factible en dimensión finita están dados respectivamente por

$A^{F}(\omega):=\left\{z^{F} \in Z^{F}: \Sigma_{i} x_{i}^{F} \leq \Sigma_{j} y_{j}^{F}+\omega\right\} \subset A(\omega)$

$P E^{F}=\left\{\left(p^{F}, z^{F}\right) \in S^{F} \times Z^{F}: p^{F} \in \cap_{j=1}^{n}\left(\varphi_{j}^{F}\left(z^{F}\right) \cap F \backslash 0\right)\right\}$

$A P E^{F}=\left\{\left(p^{F}, z^{F}\right) \in S^{F} \times A^{F}(\omega): p^{F} \in \cap_{j=1}^{n}\left(\varphi_{j}^{F}\left(z^{F}\right) \cap F \backslash 0\right)\right\}$

Por su parte las preferencias $\precsim^{F}{ }_{(i}, z^{F}{ }_{-i)}$ es el preorden inducido sobre $X_{i}\left(z^{F}{ }_{-i}\right) \cap F$ por $\precsim_{(i}, z^{F}{ }_{-i)}$

En último término recordemos que las topologías $\sigma\left(L, L^{*}\right)$ y $\sigma\left(L^{*}, L\right)$ sobre $L$ y $L^{*}$ respectivamente son, en esos espacios, las topologías más débiles que además hacen que dichos espacios sean de Hausdorff (Dunford y Schwarz, 1958, pp. 68). Al restringir $L$ a $F$ y $L^{*}$ a $F^{*}$, tenemos los subespacios $\left(F, \sigma\left(L, L^{*}\right.\right.$ )$\left._{F}\right)$ y $\left(F^{*}, \sigma\left(L^{*}, L\right)_{F^{*}}\right)$ siendo $\sigma\left(L, L^{*}\right)_{F}$ y $\sigma\left(L^{*}, L\right)_{F^{*}}$ las topologías relativas. Debemos tener presente que la restricción de $\sigma\left(L, L^{*}\right)\left(\sigma\left(L^{*}, L\right)\right)$ a $F\left(F^{*}\right)$ coincide con la restricción de la topología $\mathfrak{I}\left(\mathfrak{J}^{*}\right)^{15}$ a $F\left(F^{*}\right)$ (Zame, 1987, pp. 1095). Por este motivo, una red en $F\left(F^{*}\right) \sigma\left(L, L^{*}\right)$-converge $\left(\sigma\left(L^{*}, L\right)\right.$ -

\footnotetext{
${ }^{13} z^{F r}{ }_{-i}$ está en $\left(L \cap F_{r+k}\right)^{m+n-1}$ completando con ceros en las dimensiones $k$.

${ }^{14}$ Notemos que, como hemos dicho, esto contraste con el caso en donde no se consideran externalidades. Allí $X_{i}^{F} \subset X_{i}^{F r+k}$ siempre. Estamos hablando de aquellos trabajos que siguen la metodología de Bewley (1972).

${ }^{15}$ Recordemos que $\mathfrak{I}$ y $\mathfrak{J}^{*}$ son, respectivamente, las topologías métricas (inducidas por la norma) de $L$ y $L^{*}$.
} 
converge) si y solo si $\mathfrak{I}$-converge $\left(\mathfrak{I}^{*}\right.$-converge). Por otra parte el hecho de que la topología inducida $\sigma\left(L, L^{*}\right)_{F}$ coincida con la topología de la norma $\mathfrak{\Im}_{F}$ en dimensión finita significa que el subespacio topológico $\left(F, \sigma\left(L, L^{*}\right)_{F}\right)$ es metrizable, de ahí que podamos tratar en lugar de redes directamente con sucesiones en cada subeconomía ${ }^{16}$.

\section{TEOREMA DE EXISTENCIA, LEMAS Y SUPUESTOS ADICIONALES}

Antes de pasar al teorema central de este capítulo exponemos un supuesto y dos lemas

Supuesto PR (sobre la conducta de los productores)

(i) La correspondencia $\varphi_{j}: Z \rightarrow 2^{S}$ es valorada no vacía y convexa.

(ii) Para cada $F \in F$ la correspondencia $\varphi_{j}^{F}$ tiene un grafo cerrado

(iii) Sea $\left(z^{F(t)}, \pi^{F(t)}\right) \in Z \times S$ una subred tal que se verifican:

_ $\left(z^{F(t)}, \pi^{F(t)}\right) \rightarrow\left(z^{*}, \pi^{*}\right)$ en la topología producto débil

$\pi^{F(t)} \in \varphi_{j}\left(z^{F(t)}\right) \forall t \in T$.

$\pi^{F(t)}\left(y_{j}^{F(t)}\right)$ converge

entonces suponemos que (a) $\lim \pi^{F(t)}\left(y_{j}^{F(t)}\right) \geq \pi^{*}\left(y^{*}\right)$, y si $\lim \pi^{F(t)}\left(y_{j}^{F(t)}\right)=\pi^{*}\left(y_{j}{ }^{*}\right)$ entonces (b) $y_{j}{ }^{*} \in \partial_{\infty} Y_{j}\left(z_{-j}^{*}\right)$ y $\pi^{*} \in \varphi_{j}\left(z^{*}\right)$ para todo $j=1, \ldots, n$.

En el caso de que no hubiere externalidades o bien que estas sean fijas, este supuesto se traduce automáticamente en el supuesto PR de Bonnisseau y Meddeb (1999), por lo que así presentado es una generalización del de estos autores. Más aún, el hecho de exigir que la subred $\left(z^{F(t)}, \pi^{F(t)}\right)$ pertenezca a $Z \times S$ es más débil que el en el caso de los autores mencionados. Por otro lado, esta correspondencia extiende a $\varphi_{j}$ en Bonnisseau (1997) al espacio de bienes de infinitas dimensiones. Cuando $Y_{j}\left(z_{-j}\right)$ es un conjunto convexo entonces el supuesto PR se cumple directamente ${ }^{17}$.

\footnotetext{
${ }^{16}$ Lo mismo puede decirse del subespacio $\left(F^{*}, \sigma\left(L^{*}, L\right)_{F^{*}}\right)$

${ }^{17}$ Este tema lo desarrollamos en el apartado sobre Casos Particulares.
} 
La economía $\varepsilon^{F}$ satisface los supuestos (P), (B), (BL), (WSA), (PR), (R) y (C) (excepto NS) del trabajo de Bonnisseau (1997) para todo $F \in F$. Sin embargo, como veremos en el apéndice A, no en todas las subeconomías de dimensión finita se satisfacen los supuestos (NS) y (SA) de Bonnisseau (1997). Concretamente es a partir de un cierto $F_{0} \in F$ que ambas condiciones tienen lugar, como reza el lema siguiente.

Lema 4.1. Bajo los supuestos (C), (P), (B), (BL), (WSA) y (PR) y teniendo en cuenta lo expresado en la sección 3 , existe un subespacio $F_{0} \in F$ tal que para todo $F \in F$ con $F \supset F_{0}$ la economía $\varepsilon^{F}=\left(\left(X_{i}^{F}, \precsim_{(i}^{F}, z^{F}{ }_{-i)}, r_{i}^{F}\right)_{i=1}{ }^{m},\left(Y_{j}^{F}, \varphi_{j}^{F}\right)_{j=1}{ }^{n}\right.$, $\omega)_{F \in F}$ satisface:

$\left(\mathrm{NS}^{F}\right)$ : Sea $\left(\left(x_{i}^{F}\right)_{i=1}{ }^{m},\left(y_{j}^{F}\right)_{j=1}{ }^{n}\right) \in A^{F}(\omega)$ entonces para todo $\mathfrak{I}$-entorno de $x_{i}^{F}$, $V\left(x_{i}^{F}\right)$, y para todo i tenemos que $P_{i}^{F}\left(x_{i}^{F}\right) \cap V\left(x_{i}^{F}\right) \neq \varnothing$.

$\left(\mathrm{WSA}^{F}\right): \operatorname{Sea}\left(p^{F}, z^{F}, \lambda\right) \in P E^{F} \times \mathbb{R}_{+}, \operatorname{si} z^{F} \in A^{F}\left(\omega+\lambda \chi_{M}\right)$ entonces $p^{F}\left(\Sigma_{j} y_{j}+\omega\right.$ $\left.+\lambda \chi_{M}\right)>0$

Demostración: en el apéndice A.

Lema 4.2. Bajo los supuestos (C), (P), (B), (BL), (WSA) y (PR) y teniendo en cuenta lo expresado en la sección 3 , existe un subespacio $F_{0} \in F$ tal que para todo $F \in F$ con $F \supset F_{0}$ la economía $\varepsilon^{F}$ tiene un equilibrio $\left(z^{F}, p^{F}\right)=\left(\left(x_{i}^{F}\right)_{i=1}{ }^{m}\right.$, $\left.\left(y_{j}^{F}\right)_{j=1}^{n}, p^{F}\right) \in Z^{F} \times S^{F}$.

Demostración: en el apéndice A.

Teorema 4.1. La economía $\varepsilon=\left(\left(X_{i}, \precsim_{(i}, z_{-i)}, r_{i}\right)_{i=1}^{m},\left(Y_{j},\left(\varphi_{j}\right)_{j=1}^{n}, \omega\right)\right.$ tiene un equilibrio si satisface los supuestos (C), (P), (B), (BL), (WSA) y (PR).

La demostración la brindamos en la sección siguiente. 


\section{PASO DE LA ECONOMIA DE DIMENSION FINITA, POR LIMITE, A LA DE DIMENSION INFINITA.}

Sea $\left(\left(x_{i}^{F}\right)_{i=1}^{m},\left(y_{j}^{F}\right)_{j=1}^{n},\left(p^{F}\right)\right)$ un equilibrio de la economía $\varepsilon^{F}$ para cada $F \in \mathcal{F}$ tal que $F \supset F_{0}$. Probaremos la existencia de un vector $\left(\left(x_{i}^{*}\right)_{i=1}{ }^{m},\left(y_{j}{ }^{*}\right)_{j=1}{ }^{n}\right.$, $\left.\left(\pi^{*}\right)\right) \in L^{m+n} \times S^{n}$, el cual es un equilibrio de la economía. La demostración consta de los siguientes 9 pasos:

Paso 1. Extensión de $p^{F}\left(p^{F}: L \cap F \mapsto \mathbb{R}_{+}\right)$a $\pi^{F}\left(\pi^{F}: L \mapsto \mathbb{R}_{+}\right)$en $S$.

\section{Demostración}

La demostración no es sino una aplicación del teorema de HahnBanach (Kolmogorov y Fomin, 1957, pp. 86). Existe una extensión $\pi^{F}$ de $p^{F}$ a $L$. Como $p^{F} \in S^{F}$, que es un conjunto compacto, $\left\|p^{F}\right\|<\infty$. En virtud del teorema mencionado $\left\|\pi^{F}\right\|_{L^{*}}=\left\|p^{F}\right\|<\infty$. Como $\pi^{F}\left(\chi_{M}\right)=p^{F}\left(\chi_{M}\right)=1$, entonces para toda $F \in F \pi^{F} \in S$.

Observación. Notemos que en virtud de lo que expusimos en la sección 3 acerca de la correspondencia $\varphi_{j}^{F}$ sabemos que para cada $p^{F} \in \varphi_{j}^{F}\left(z^{F}\right)$ existirá una extensión $p_{j}^{F} \in \varphi_{j}\left(z^{F}\right)$. Es decir $p_{j \mid F}^{F}=p^{F}$. No obstante no tenemos ningún argumento para afirmar ni que $p_{j}^{F}$ es igual para toda $j$ (aunque sí lo sea su restricción a $F$ ), ni que para alguna $j p_{j}^{F}=\pi^{F}$.

Paso 2. Existe una subred $\left(\left(x_{i}^{F(t)}\right)_{i=1}^{m},\left(y_{j}^{F(t)}\right)_{j=1}{ }^{n}, \pi^{F(t)},\left(p_{j}^{F(t)}\right)\right)_{t \in(T, \geq)}$ que converge en la topología producto débil a $\left(\left(x_{i}^{*}\right)_{i=1}{ }^{m},\left(y_{j}^{*}\right)_{j=1}{ }^{n}, \pi^{*},\left(p_{j}^{*}\right)\right)$. Del mismo modo $\left(\pi^{F(t)}\left(y_{j}^{F(t)}\right)\right)_{t \in(T, \geq)}, j=1, \ldots, n \mathrm{y}\left(\pi^{F(t)}\left(x_{i}^{F(t)}\right)\right)_{t \in(T, \geq)}, i=1, \ldots m$ son redes convergentes.

\section{Demostración}

En primer lugar probamos que la red $\left(\left(x_{i}^{F}\right)_{i=1}^{m},\left(y_{j}^{F}\right)_{j=1}^{n}\right)_{F \in \mathcal{F}}$ está en un conjunto débilmente compacto. En efecto $\left(\left(x_{i}^{F}\right)_{i=1}{ }^{m},\left(y_{j}^{F}\right)_{j=1}{ }^{n}\right)_{F \in F} \in A^{\prime}(\omega)$ que según $\mathrm{B}$ es un conjunto acotado en la norma, en consecuencia por el teorema de Banach-Alaoglu (Ash, 1972, pp. 162) $\left(\left(x_{i}^{F}\right)_{i=1}^{m},\left(y_{j}^{F}\right)_{j=1}{ }^{n}\right)_{F \in F}$ está en un conjunto débilmente ${ }^{*}$ compacto. Así existe una subred $\left(\left(x_{i}^{F(t)}\right)_{i=1}{ }^{m},\left(y_{j}^{F(t)}\right)_{j=1}{ }^{n}\right)_{t \in(T, \geq)}$ convergente en la topología producto a $\left(\left(x_{i}{ }^{*}\right)_{i=1}{ }^{m},\left(y_{j}{ }^{*}\right)_{j=1}{ }^{n}\right)$. 
Análogamente, del paso 1 tenemos que las redes $\left(\pi^{F}\right)_{F \in F} \mathrm{y}\left(\left(p_{j}^{F}\right)\right)_{F \in F}$ están en el conjunto $S$, y nuevamente por el teorema de Banach-Alaoglu, este es débilmente ${ }^{*}$ compacto. Existen entonces las subredes $\left(\pi^{F(t)}\right)_{t \in(T, \geq)}$ y $\left(\left(p_{j}^{F(t)}\right)\right)_{t \in(T,}$ য) $\operatorname{con} \pi^{F(t) w^{*}} \rightarrow \pi^{*}$ y $p_{j}^{F(t) w^{*}} \rightarrow p_{j}^{*}$ para $j=1,2, \ldots, n$. Entonces la red $\left(\left(x_{i}^{F(t)}\right)_{i=1}{ }^{m}\right.$, $\left.\left(y_{j}^{F(t)}\right)_{j=1}{ }^{n}, \pi^{F(t)},\left(p_{j}^{F(t)}\right)\right)_{t \in(T, \geq)}$ converge en la topología débil a $\left(\left(x_{i}^{*}\right)_{i=1}{ }^{m},\left(y_{j}^{*}\right)_{j=1}{ }^{n}\right.$, $\left.\pi^{*},\left(p_{j}^{*}\right)\right)$.

Por un análisis similar tendríamos que $\pi^{F}\left(y_{j}^{F}\right) \in \mathbb{R}^{n}=\mathbb{R}^{n^{*}}$ y $\left|\pi^{F}\left(y_{j}^{F}\right)\right|$ $\leq^{18}\left\|\pi^{F}\right\|_{b a} .\left\|\left(y_{j}^{F}\right)\right\|_{L_{\infty}}<\infty$. Análogo a lo que expusimos arriba podemos afirmar que la subred $\left(\pi^{F(t)}\left(y_{j}^{F(t)}\right)\right)_{t \in(T, \geq)}$ converge para toda $j$. La demostración para $\left(\pi^{F(t)}\left(x_{i}^{F(t)}\right)\right)_{t \in(T, \geq)}, i=1, \ldots m$ es idéntica $\mathbf{\square}$.

Observación 1. El paso anterior es equivalente a decir que las redes $\left(p_{j}^{F(t)}\left(y_{j}^{F(t)}\right)\right)_{t \in(T, \geq)}, j=1, \ldots, n \mathrm{y}\left(p_{j}^{F(t)}\left(x_{i}^{F(t)}\right)\right)_{t \in(T, \geq)}, i=1, \ldots m$, son convergentes puesto que restringidas a $F(t)$, para todo $t \in T, p^{F(t)}, p_{j}^{F(t)}$ y $\pi^{F(t)}$ coinciden. Observemos también que la convergencia aquí es en la topología inducida por la norma.

Observación 2. Notemos que $\left(\left(x_{i}^{F(t)}\right)_{i=1}{ }^{m},\left(y_{j}^{F(t)}\right)_{j=1}{ }^{n}, p^{F(t)}\right)_{t \in(T, \geq)}$ es un subconjunto del conjunto de equilibrios $\left(\left(x_{i}^{F}\right)_{i=1}{ }^{m},\left(y_{j}^{F}\right)_{j=1}{ }^{n},\left(p^{F}\right)\right)_{F \in F}$, por lo que para cada $t \in$ $T$ con $t$ mayor que un cierto $t_{0} \in T^{19}\left(\left(x_{i}^{F(t)}\right)_{i=1}^{m},\left(y_{j}^{F(t)}\right)_{j=1}{ }^{n},\left(p^{F(t)}\right)\right)$ es un equilibrio de $\varepsilon^{F(t)}$.

Paso 3. Para todo $j=1, \ldots, n, \pi^{*}=p_{j}^{*}$.

Demostración. 297.

La prueba es similar a la dada por Bonnisseau y Meddeb (1999), pp.

Paso 4. $\left(\left(x_{i}^{*}\right)_{i=1}^{m},\left(y_{j}{ }^{*}\right)_{j=1}{ }^{n}\right) \in \prod_{i=1}{ }^{m} X_{i}\left(z_{-i}^{*}\right) \times \prod_{j=1}^{n} Y_{j}\left(z_{-j}^{*}\right)$ y $\sum_{i=1}^{m} x_{i}^{*}=\sum_{j=1}^{n} y_{j}^{*}+$ $\omega$.

\footnotetext{
${ }^{18}$ Esta desigualdad es ampliamente conocida y puede consultarse en Vulikh, B. (1963) pp. 219.

${ }^{19} \mathrm{Y}$ esto en virtud del lema 4.2, pues para $t_{0} \in T$, podemos hacer $F\left(t_{0}\right)=F_{0}$.
} 


\section{Demostración.}

Del paso 2 observación $2\left(\left(x_{i}^{F(t)}\right)_{i=1}^{m},\left(y_{j}^{F(t)}\right)_{j=1}{ }^{n}\right) \in \mathrm{Z}^{F(t)} \subset \prod_{i=1}^{m} X_{i}\left(z^{F(t)}\right.$. $\left.{ }_{i}\right) \times \prod_{j=1}^{n} \partial_{\infty} Y_{j}\left(z^{F(t)}{ }_{j}\right) \subset \prod_{i=1}^{m} X_{i}\left(z^{F(t)}{ }_{-i}\right) \times \prod_{j=1}^{n} Y_{j}\left(z^{F(t)}{ }_{-j}\right)$ para todo $t>t_{0}, t \in T$. Como $\mathrm{z}^{F(t)}{ }^{w} \rightarrow \mathrm{z}^{*}$, y las correspondencias $X_{i}$ e $Y_{j}$ son débilmente cerradas entonces $\left(\left(x_{i}^{*}\right)_{i=1}{ }^{m},\left(y_{j}{ }^{*}\right)_{j=1}{ }^{n}\right) \in \prod_{i=1}^{m} X_{i}\left(z_{-i}^{*}\right) \times \prod_{j=1}{ }^{n} Y_{j}\left(z_{-j}^{*}\right)$. Por otra parte $\sum_{i=1}{ }^{m} x_{i}{ }^{F(t)}=\sum$ ${ }_{j=1}^{n} y_{j}{ }^{F(t)}+\omega$ a partir de un cierto $t_{0} \in T$. Luego en virtud de las $\sigma\left(L, L^{*}\right)$ convergencias de $x_{i}^{F(t)}$ y de $y_{j}^{F(t)}$ tenemos que $\sum_{i=1}^{m} x_{i}{ }^{*}=\sum_{j=1}^{n} y_{j}{ }^{*}+\omega$.

Paso 5. $\pi^{*} \neq 0$.

\section{Demostración.}

$\pi^{*} \geq 0\left(\pi^{*} \in L^{*}{ }_{+}\right) . \quad \forall t \in T, \pi^{F(t)}\left(\chi_{M}\right)=1$. Entonces $1=\lim \pi^{F(t)}\left(\chi_{M}\right)=\pi^{*}\left(\chi_{M}\right)$. Luego $\pi^{*}=p_{j}^{*} \neq 0 \forall j=1, \ldots, n$

Paso 6. Si : $x_{i} \succsim_{\left(i, z^{*}{ }_{-i)}\right.} x_{i}^{*}$ entonces $\pi^{*}\left(x_{i}\right) \geq r_{i}\left(\pi^{*}\left(\omega_{i}\right), \lim \left(\pi^{F(t)}\left(y_{j}^{F(t)}\right)\right)\right)$

\section{Demostración.}

Sean $x_{i}, x_{i}{ }^{*} \in X_{i}\left(z_{-i}^{*}\right)$ con $x_{i} \succsim_{(i,}, z^{*}{ }_{-i)} x_{i}{ }^{*}$ y sea $y_{j}{ }^{*} \in \partial_{\infty} Y_{j}\left(z_{-j}^{*}\right)$. Por el supuesto de no saciedad local tenemos a $x_{i}^{\prime} \in P_{i}\left(x_{i}\right) \cap V\left(x_{i}\right)$, donde $V\left(x_{i}\right)$ es un $\sigma\left(L, L^{*}\right)$-entorno de $x_{i}$. Ahora bien, $x_{i}{ }^{\prime}$ está en algún $F \in F$ y existe $t_{0} \in T$ tal que para todo $t>t_{0} F \subset F(t)$, de modo que $x_{i}{ }^{\prime} \in P_{i}\left(x_{i}\right) \cap V\left(x_{i}\right) \cap F(t) \forall t>t_{0}$. Es evidente que $x_{i}{ }^{\prime} \in X_{i}\left(z^{*}{ }_{-i}\right) \cap F\left(t: t>t_{0}\right) \cap P_{i}\left(x_{i}\right) \cap V\left(x_{i}\right)$ y por C(ii) existe $t_{1} \in T$ tal que para todo $t>t_{1} x_{i}{ }^{\prime} \in X_{i}\left(z^{F(t: t>t 1)}{ }_{-i}\right) \cap F\left(t: t>t_{0}\right) \cap P_{i}\left(x_{i}\right) \cap V\left(x_{i}\right)$. Entonces para todo $t \in T$ con $t>$ máx. $\left\{t_{0}, t_{1}\right\} x_{i}{ }^{\prime} \in X_{i}\left(z^{F(t)}{ }_{-i}\right) \cap F(t) \cap P_{i}\left(x_{i}\right) \cap V\left(x_{i}\right)$. Por otro lado $x_{i}^{F(t) w^{*}} \rightarrow x_{i}^{*}$ y $x_{i}^{F(t)} \in X_{i}\left(z^{F(t)}{ }_{-i}\right) \cap F(t)$ para todo $t \in T$. Así en particular, existe $t_{2}$ $\in T$ tal que para todo $t>t_{2} x_{i}^{F(t)} \in X_{i}\left(z^{F(t)}{ }_{-i}\right) \cap F(t) \cap U\left(x_{i}^{*}\right)$, donde $U\left(x_{i}^{*}\right)$ es un entorno de $x_{i}^{*}$. De igual manera y teniendo en cuenta $\mathrm{P}($ ii) se sigue que existe $y_{j}{ }^{\prime} \in Y_{j}\left(z_{-j}^{*}\right)$ y $t_{3} \in T$ tal que para todo $t>t_{3} y_{j}{ }^{\prime} \in Y_{j}\left(z^{F(t)}{ }_{-i}\right) \cap F(t)$. Entonces para todo $t>\max \left\{t_{0}, t_{1}, t_{2}, t_{3}\right\}, t \in T$, consideramos la economía $\varepsilon^{F(t)}$. De ahí por la continuidad de las preferencias $x_{i}^{\prime} \succ_{(i,}^{F(t)} z^{F(t)}{ }_{-i)} x_{i}^{F(t)}$ de modo que ${ }^{20}$

\footnotetext{
${ }^{20}$ Tengamos presente que estamos considerando el hecho de que $\left(\left(x_{i}^{F(t)}\right)_{i=1}^{m},\left(y_{j}^{F(t)}\right)_{j=1}{ }^{n},\left(\pi^{F(t)}\right)\right)_{t \in(T, z)}$ es un equilibrio en $\varepsilon^{F(t)}$ y que $x_{i} \succ^{F(t)}{ }_{(i,}, z^{F(t)}{ }^{-i)} x_{i}^{F(t)}$ (véase la nota al pie de página anterior).
} 
$\pi^{F(t)}\left(x_{i}^{\prime}\right)>\pi^{F(t)}\left(x_{i}^{F(t)}\right)=r_{i}\left(\pi^{F(t)}\left(\omega_{i}\right),\left(\pi^{F(t)}\left(y_{j}^{F(t)}\right)\right)\right) \geq r_{i}\left(\pi^{F(t)}\left(\omega_{i}\right),\left(\pi^{F(t)}\left(y_{j}^{\prime}\right)\right)\right)$

Tomando límites

$\pi^{*}\left(x_{i}^{\prime}\right) \geq \lim \pi^{F(t)}\left(x_{i}^{F(t)}\right)=r_{i}\left(\pi^{*}\left(\omega_{i}\right), \lim \left(\pi^{F(t)}\left(y_{j}^{F(t)}\right)\right)\right) \geq r_{i}\left(\pi^{*}\left(\omega_{i}\right),\left(\pi^{*}\left(y_{j}^{\prime}\right)\right)\right)$ (1)

Esta desigualdad vale para $x_{i}$ arbitrariamente cerca de $x_{i}$, de modo que la continuidad de $\pi^{*}$ y de $r_{i}$ aseguran que $\pi^{*}\left(x_{i}\right) \geq r_{i}\left(\pi^{*}\left(\omega_{i}\right), \lim \left(\pi^{F(t)}\left(y_{j}^{F(t)}\right)\right)\right)$ $\left(1^{\prime}\right)$

Paso 7. $\pi^{*}\left(y_{j}^{*}\right)=\lim \pi^{F(t)}\left(y_{j}^{F(t)}\right)$ y $\pi^{*}\left(x_{i}^{*}\right)=\lim \left(\pi^{F(t)}\left(x_{i}^{F(t)}\right)\right)$

En (1') sustituimos $x_{i}$ por $x_{i}{ }^{*}$ y en (1) $y_{j}$ por $y_{j}{ }^{*}$, de modo que tenemos $\pi^{*}\left(x_{i}^{*}\right) \geq \lim \pi^{F(t)}\left(x_{i}^{F(t)}\right)=r_{i}\left(\pi^{*}\left(\omega_{i}\right), \lim \left(\pi^{F(t)}\left(y_{j}^{F(t)}\right)\right)\right) \geq r_{i}\left(\pi^{*}\left(\omega_{i}\right), \quad\left(\pi^{*}\left(y_{j}^{*}\right)\right)\right)$ (2)

Veamos que $\pi^{*}\left(x_{i}^{*}\right)=r_{i}\left(\pi^{*}\left(\omega_{i}\right),\left(\pi^{*}\left(y_{j}^{*}\right)\right)\right)$ para todo $i$. Supongamos que no, entonces existe $i^{\prime}$ tal que $\pi^{*}\left(x_{i^{*}}{ }^{*}\right)>r_{i}\left(\pi^{*}\left(\omega_{i}\right),\left(\pi^{*}\left(y_{j}^{*}\right)\right)\right)$. Entonces $\sum_{i=1}{ }^{m}$ $\pi^{*}\left(x_{i}^{*}\right)>\pi^{*}(\omega)+\sum_{j=1}^{n} \pi^{*}\left(y_{j}^{*}\right)$

Por otro lado, hemos probado que $\sum_{i=1}^{m} x_{i}^{*}=\sum_{j=1}^{n} y_{j}^{*}+\omega$, lo que implica que $\sum_{i=1}^{m} \pi^{*}\left(x_{i}^{*}\right)=\pi^{*}(\omega)+\sum_{j=1}^{n} \pi^{*}\left(y_{j}^{*}\right)$. De modo que es una contradicción con (3). Luego $\pi^{*}\left(x_{i}^{*}\right)=r_{i}\left(\pi^{*}\left(\omega_{i}\right),\left(\pi^{*}\left(y_{j}^{*}\right)\right)\right)$ para todo $i=1, \ldots, m$. Entonces, de la desigualdad (2) se deduce que $r_{i}\left(\pi^{*}\left(\omega_{i}\right), \lim \left(\pi^{F(t)}\left(y_{j}^{F(t)}\right)\right)\right)=$ $r_{i}\left(\pi^{*}\left(\omega_{i}\right),\left(\pi^{*}\left(y_{j}^{*}\right)\right)\right)$ para todo $i$. Dado que $r_{i}($.$) es estrictamente creciente,$ $\left.\lim \pi^{F(t)}\left(y_{j}^{F(t)}\right)\right)=\pi^{*}\left(y_{j}^{*}\right)$. La demostración de que $\pi^{*}\left(x_{i}^{*}\right)=\lim \left(\pi^{F(t)}\left(x_{i}^{F(t)}\right)\right)$ es similar .

Notemos que con lo expuesto en este paso y considerando lo que probamos en el paso 4 junto al supuesto PR(iii) (b) tenemos que en definitiva $\left(\left(x_{i}^{*}\right)_{i=1}^{m},\left(y_{j}^{*}\right)_{j=1}{ }^{n}\right)=z^{*} \in A(\omega)$.

Paso 8. Para todo $i=1, \ldots, m, x_{i}{ }^{*}$ es maximal en el conjunto presupuestario $\left\{x_{i}\right.$ $\left.\in X_{i}\left(z_{-i}^{*}\right): \pi^{*}\left(x_{i}\right) \leq r_{i}\left(\pi^{*}\left(\omega_{i}\right),\left(\pi^{*}\left(y_{j}^{*}\right)_{j=1}{ }^{n}\right)\right)\right\}$ 


\section{Demostración:}

Tenemos que probar que para cada agente $i$, si $x_{i} \succ_{(i,}, z_{-i)}^{*} x_{i}^{*}$ entonces $\pi^{*}\left(x_{i}\right)>$ $\pi^{*}\left(x_{i}^{*}\right)$. Supongamos que $\pi^{*}\left(x_{i}^{\prime}\right)=\pi^{*}\left(x_{i}^{*}\right)$. Por los supuestos WSA y C(v) sabemos que $\pi^{*}\left(x_{i}^{*}\right)=r_{i}\left(\pi^{*}\left(\omega_{i}\right),\left(\pi^{*}\left(y_{j}^{*}\right)_{j=1}{ }^{n}\right)\right)>0$. Para todo $t \in(0,1)$ tendremos que $\pi^{*}\left(t x_{i}{ }^{\prime}\right)<\pi^{*}\left(x_{i}{ }^{\prime}\right)=\pi^{*}\left(x_{i}^{*}\right)$. Sea asimismo $t$ lo suficientemente cercano a 1 tal que $t x_{i}{ }^{\prime} \in X_{i}\left(z^{*}{ }_{-i}\right)$. Por la continuidad de las preferencias $t x_{i}{ }^{\prime} \succ_{(i}$, $z_{-i)}^{*} x_{i}^{*}$ y por el paso $7 \pi^{*}\left(t x_{i}{ }^{\prime}\right) \geq \pi^{*}\left(x_{i}^{*}\right)$. Tenemos así una contradicción.

Paso 9. $\pi^{*} \in \varphi_{j}\left(z^{*}\right)$

\section{Demostración:}

De los pasos 1 y 2 tenemos que para todo $t \in T p_{j}^{F(t)} \in \varphi_{j}\left(z^{F(t)}\right)$. Por los pasos 2 y 3 tenemos que $p_{j}^{F(t)} w^{*} \rightarrow \pi^{*} \in S$ para todo $j$ y $p_{j}^{F(t)}\left(y_{j}^{F(t)}\right)=$ $\pi^{F(t)}\left(y_{j}^{F(t)}\right)$ converge. Por su parte en el paso 7 dedujimos que $\lim \pi^{F(t)}\left(y_{j}^{F(t)}\right)=$ $\pi^{*}\left(y_{j}^{*}\right)$, entonces por $\operatorname{PR}\left(\right.$ iii) b) $\pi^{*} \in \cap_{j=1}^{n} \varphi_{j}\left(z^{*}\right)$.

\section{APENDICE A}

Incumplimiento de los supuestos (WSA) y (NS) en subeconomías de dimensión finita

En primer lugar notemos que el supuesto (WSA) no se cumple en cualquier subeconomía finita. Sean $\left(p^{F}, z^{F}, \lambda\right) \in P E^{F} \times \mathbb{R}_{+}$y $z^{F} \in A^{F}\left(\omega+\lambda \chi_{M}\right)$ entonces en virtud de que $\mathrm{Z}^{F} \subset \mathrm{Z}$ tenemos que en realidad $\left(p^{F}, z^{F}, \lambda\right) \in P E \times \mathbb{R}_{+}$ y $z^{F} \in A\left(\omega+\lambda \chi_{M}\right)$. Del punto 5 paso 1 tenemos que existe $p_{j}^{F} \in \varphi_{j}\left(z^{F}\right)$ tal que $p_{j \mid F}^{F}=\pi_{\mid F}^{F}=p^{F}$. Pero si bien es cierto que $\left(p^{F}\right) \in \prod_{i=1}^{m} \varphi_{j}^{F}\left(z^{F}\right)$ ya hemos visto que la extensión de $p_{j}^{F}$ a $L$ no ha de ser necesariamente la misma para todo $j$, de modo que lo que en definitiva tendremos es que $\left(p_{j}\right) \in \Pi_{j=1}^{n} \varphi_{j}\left(z^{F}\right)$ por lo que no podríamos hacer uso del supuesto (WSA).

El caso del supuesto de no saciedad local (NS) ocurre que si $\left(z^{F}\right) \in$ $A^{F}(\omega)$ entonces $\left(z^{F}\right) \in A(\omega)$. Por (NS) sabemos que en cualquier $\sigma$-entorno de $x_{i}^{F}$ habrá un $x_{i}{ }^{\prime}$ tal que $x_{i}{ }^{\prime} \succ{ }_{(i}, z^{F}{ }_{-i)} x_{i}^{F}$. Sin embargo no podemos afirmar que esté en el subespacio $F$, de ahí que no podamos sugerir que se satisfaga la condición (NS) en cualquier subeconomía $\varepsilon^{F}$. 
En el siguiente paso demostramos que estos supuestos se cumplen para economía finitas a partir de una cierta dimensión.

\section{Demostración del lema 4.1}

$\left(\mathrm{WSA}^{F}\right)$

En primer lugar probamos que para todo $F \supset F_{0}$ la economía $\varepsilon^{F}$ satisface el supuesto $\left(\mathrm{WSA}^{F}\right)$. Lo demostramos por reducción al absurdo. En efecto si suponemos que no, entonces para todo $F \in F$ existe $F^{\prime}, F^{\prime} \supset F$, tal que si $\left(p^{F^{\prime}}, z^{F^{\prime}}, \lambda\right) \in P E^{F^{\prime}} \times \mathbb{R}_{+}$y $z^{F^{\prime}} \in A^{F^{\prime}}\left(\omega+\lambda \chi_{M}\right)$ entonces $p^{F^{\prime}}\left(\Sigma_{j} y_{j}+\omega+\lambda \chi_{M}\right)$ $=0$. Esta demostración es similar a la de Bonnisseau (2000), sin embargo allí $\lambda$ está en un intervalo cerrado de $\mathbb{R}_{+}$, en tanto que aquí $\lambda \in \mathbb{R}_{+}$y la hemos adaptado al hecho de que estamos trabajando con correspondencias. Del paso 1 en la sección $\mathrm{V}$ tenemos que existe $p_{j} F^{\prime} \in \varphi_{j}\left(z^{F^{\prime}}\right)$ tal que $p_{j \mid F^{\prime}}{ }^{F^{\prime}}=\pi_{\mid F^{\prime}}{ }^{F^{\prime}}=p^{F^{\prime}}$. Sabemos que la red $\left(\left(z^{F^{\prime}}\right), \pi^{F^{\prime}},\left(p_{j}{ }^{F^{\prime}}\right),\left(p_{j}^{F^{\prime}}\left(y_{j}{ }^{F^{\prime}}\right)\right)_{F^{\prime} \in F}\right.$ está contenido en un conjunto débilmente compacto. Entonces existe una subred $\left(\left(z^{F^{\prime}(t)}\right), \pi^{F^{\prime}(t)},\left(p_{j}^{F}\right.\right.$ $\left.\left.{ }^{\prime}(t)\right),\left(p_{j}^{F^{\prime}(t)}\left(y_{j}^{F^{\prime}(t)}\right)\right)\right)_{t \in(T, \geq)}$ convergente a $\left(\left(z^{*}\right), \pi^{*},\left(p_{j}^{*}\right), \lim \left(p_{j}^{F^{\prime}(t)}\left(y_{j}^{F^{\prime}(t)}\right)\right)\right)$.

Como $z^{F^{\prime}} \in A^{F^{\prime}}\left(\omega+\lambda \chi_{M}\right)$ para todo $F^{\prime} \in F$, deducimos que $\sum_{j=1}^{n} y_{j}^{F^{\prime}(t)}$ $+\omega+\lambda \chi_{M} \geq \sum_{i=1}{ }^{m} x_{i}^{F^{\prime}(t)} \geq 0$, y como $L_{+}$es débilmente cerrado $\sum_{j=1}^{n} y_{j}^{*}+\omega+$ $\lambda \chi_{M} \geq \sum_{i=1}^{m} x_{i}^{*} \geq 0$. Además tengamos presente que $\pi^{*}=p_{j}^{*}$ para todo $j$ (sección V paso 3). Como $\pi^{*} \geq 0, \sum_{j=1}^{n} \pi^{*}\left(y_{j}^{*}\right)+\pi^{*}(\omega)+\lambda \geq 0$. Por otra parte, dado que $p^{F^{\prime}}\left(\Sigma_{j} y_{j}+\omega+\lambda \chi_{M}\right)=0$ para todo $F^{\prime} \in F$, entonces deducimos que $\sum_{j=1}{ }^{n} \lim \pi^{F^{\prime}(t)}\left(y_{j}^{F^{\prime}(t)}\right)+\pi^{*}(\omega)+\lambda=0$. Por el supuesto PR (iii) a), $0 \leq \sum_{j=1}^{n}$ $\pi^{*}\left(y_{j}^{*}\right)+\pi^{*}(\omega)+\lambda \leq \sum_{j=1}{ }^{n} \lim \pi^{F^{\prime}(t)}\left(y_{j}^{F^{\prime}(t)}\right)+\pi^{*}(\omega)+\lambda=0$, de donde se deduce que
$\sum{ }_{j=1}^{n}$
$\pi^{*}\left(y_{j}^{*}\right)$
$\pi^{*}(\omega)+$
$\lambda \quad=$
0

$\mathrm{y}$

$\sum_{j=1}^{n} \pi^{*}\left(y_{j}^{*}\right)=\sum_{j=1}{ }^{n} \lim \pi^{F^{\prime}(t)}\left(y_{j}^{F^{\prime}(t)}\right)$, o sea $\pi^{*}\left(y_{j}^{*}\right)=\lim \pi^{F^{\prime}(t)}\left(y_{j}^{F^{\prime}(t)}\right)$ para toda $j$.

Tengamos presente lo siguiente: en primer lugar $\pi^{*}=p_{j}^{*}$ y $p_{j \mid F^{\prime}(t)} F^{F^{\prime}(t)}=$ $\pi_{\mid F^{\prime}(t)}^{F^{\prime}(t)}$ para todo $j$. En segundo término, dado que $z^{F^{\prime}} \in Z^{F^{\prime}}$ para todo $F^{\prime} \in \mathcal{F}$, entonces $y_{j}^{F^{\prime}(t)} \in \partial_{\infty} Y_{j}\left(z^{F^{\prime}(t)}{ }_{-j}\right) \forall j, t$, y $x_{i}^{F^{\prime}(t)} \in X_{i}\left(z^{F^{\prime}(t)}{ }_{-i}\right) \forall i$, t. Como la 
correspondencias $X_{i}$ e $Y_{j}$ son cerradas (ambas en la topología débil) y como $\pi^{*}\left(y_{j}^{*}\right)=\lim \pi^{F^{\prime}(t)}\left(y_{j}^{F^{\prime}(t)}\right)$ para toda $j$, entonces por el supuesto PR(iii) b) $\pi^{*} \in$ $\varphi_{j}\left(z^{*}\right)$ para todo $j=1, \ldots, n \mathrm{y} z^{*} \in Z$.

De ahí que $\left(\pi^{*}, z^{*}, \lambda\right) P E \times \mathbb{R}_{+}$y $z^{*} \in A\left(\omega+\lambda \chi_{M}\right)$ entonces por el supuesto WSA $\sum_{j=1}^{n} \pi^{*}\left(y_{j}^{*}\right)+\pi^{*}(\omega)+\lambda>0$, lo cual es una contradicción con (1). Luego existe $F_{0} \in F$ tal que para todo $F \in F \operatorname{con} F \supset F_{0}$ la economía $\varepsilon^{F}$ satisface $\left(\mathrm{WSA}^{F}\right)$

$\left(\mathrm{NS}^{F}\right)$

A continuación probamos que se satisface también $\left(\mathrm{NS}^{F}\right)$ a partir de un cierto $F_{0} \in F$. La demostración dada es similar a la desarrollada por Bonnisseau (2000), sin embargo en nuestro caso esta se ha extendido al considerar externalidades y correspondencias.

Supongamos que no existe tal $F_{0} \in \mathcal{F}$, entonces para todo $F \in F$ existe $F^{\prime}, F^{\prime} \supset F$, tal que si $\left(\left(x_{i}^{F^{\prime}}\right)_{i=1}{ }^{m},\left(y_{j}^{F^{\prime}}\right)_{j=1}{ }^{n}\right) \in A^{F^{\prime}}(\omega)$ y para cualquier $\mathfrak{I}$-entorno de $x_{i}^{F^{\prime}}, V\left(x_{i}^{F^{\prime}}\right), i=1, \ldots, n$; entonces hay algún $i, i_{0}$, donde $P_{i 0}{ }^{F}\left(x_{i 0}{ }^{F}\right) \cap V\left(x_{i 0}{ }^{F}\right)=$ $\varnothing$. De B y de los pasos 2, 4 y 7 de la sección $\mathrm{V}$ sabemos que existe una subred $\left(\left(x_{i}^{F^{\prime}(t)}\right)_{i=1}^{m},\left(y_{j}^{F^{\prime}(t)}\right)_{j=1}{ }^{n}\right){ }_{t \in(T, \geq)}$ convergente a $\left(\left(x_{i}^{*}\right)_{i=1}{ }^{m},\left(y_{j}^{*}\right)_{j=1}{ }^{n}\right) \in A(\omega)$. Del supuesto C(iii) sabemos que existe $\zeta_{i} \in P_{i}\left(x_{i}{ }^{*}\right) \cap V\left(x_{i}^{*}\right)$ para todo $i=1, \ldots, m, \mathrm{y}$ todo $\sigma$-entorno, $V\left(x_{i}{ }^{*}\right)$,de $x_{i}{ }^{*}$. De nuevo, por C(iii) $P_{i}\left(\zeta_{i}\right) \cap V\left(\zeta_{i}\right) \neq \varnothing$.

Sea $\zeta_{i}{ }^{\prime} \in P_{i}\left(\zeta_{i}\right) \cap V\left(\zeta_{i}\right), \zeta_{i}{ }^{\prime} \in F \subset F(t)$ para todo $t>t_{0}$, entonces $P_{i}\left(\zeta_{i}\right) \cap V\left(\zeta_{i}\right) \cap F(t) \neq \varnothing$. Para todo $i$ tenemos entonces claramente que $X_{i}\left(z^{*}\right.$. $\left.{ }_{i}\right) \cap P_{i}\left(\zeta_{i}\right) \cap V\left(\zeta_{i}\right) \cap F(t) \neq \varnothing$ entonces por C(ii) existe $t_{1}$ tal que si $t>t_{1}, X_{i}\left(z^{F^{\prime}(t)}\right.$. $\left.{ }_{i}\right) \cap P_{i}\left(\zeta_{i}\right) \cap V\left(\zeta_{i}\right) \cap F(t) \neq \varnothing$ o bien para todo $t>\max \left\{t_{0}, t_{1}\right\} X_{i}^{F^{\prime}(t)}\left(z^{F^{\prime}(t)}\right.$. $\left.{ }_{i}\right) \cap P_{i}\left(\zeta_{i}\right) \cap V\left(\zeta_{i}\right) \neq \varnothing$.

Por otra parte, sabemos que para todo $t \in T, x_{i}^{F^{\prime}(t)} \in X_{i}^{F^{\prime}(t)}\left(z^{F^{\prime}(t)}{ }_{-i}\right)$ y como la red $\left(x_{i}^{F^{\prime}(t)}\right)_{t \in(T, \geq)}$ es débilmente convergente, existe $t_{2}$ tal que si $t>t_{2}$, $x_{i}^{F^{\prime}(t)} \in X_{i}^{F^{\prime}(t)}\left(z^{F^{\prime}(t)}{ }_{-i}\right) \cap U\left(x_{i}^{*}\right)$ donde $U\left(x_{i}^{*}\right)$ es un $\sigma$-entorno de $x_{i}^{*}$. Entonces para todo $t>\max \left\{t_{0}, t_{1}, t_{2}\right\} \zeta_{i}{ }^{\prime}$ y $x_{i}{ }^{{ }^{\prime}(t)}$ pertenecen a $X_{i}^{F(t)}\left(z^{F(t)}{ }_{i}\right)$ y, por la continuidad de las preferencias, para cualquier $i=1, \ldots, m, \zeta_{i} \succ^{F^{\prime}(t)}{ }_{(i,}, z^{F^{\prime}(t)}{ }_{-i)} x_{i}^{F}$ '(t) 21 . Ahora bien, $\left\{F^{\prime}(t): t \in T\right\}$ es un subconjunto del conjunto $\{F: F \in F\}$ por lo que la preferencia estricta de arriba contradice que para cualquier $F^{\prime} \in F$

\footnotetext{
${ }^{21}$ Aquí $\zeta_{i}{ }^{\prime}$ no es necesariamente el mismo vector que en el párrafo anterior.
} 


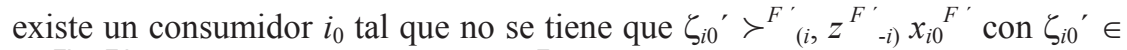
$X_{i 0} F^{\prime}\left(z^{F^{\prime}}-i 0\right)$. Luego, la condición $\left(\mathrm{NS}^{F}\right)$ se cumple a partir de un cierto $F_{0} \in F$.

\section{APENDICE B}

Casos Particulares

En este apartado veremos tres casos particulares: 1) El espacio de bienes es de dimensión finita, 2) No existen externalidades o bien estas son fijas y 3) Los conjuntos de producción son convexos. El propósito de esta sección es mostrar como el teorema de existencia 4.1 extiende a los de otros trabajos en donde se dan una o varias de las circunstancias expuestas a continuación.

\section{1) Economías con espacio de bienes de dimensión finita}

Este caso ya lo hemos analizado implícitamente en la sección dedicada al estudio de las subeconomías o economías truncadas. Allí observamos la validación de los conjuntos $X_{i}^{F}, Y_{j}^{F}, Z^{F}, A^{F}(\omega)$ y $\varphi_{j}^{F}$ entre otros. En el apéndice B probamos que cuando el espacio de bienes es a partir de una cierta dimensión -finita- se cumplen todos los supuestos de Bonnisseau (1997), además es posible seguir la misma argumentación en la prueba de demostración que lleva adelante este autor, adaptándola a cualquier espacio de dimensión finita como es nuestro caso.

2) No existen externalidades o bien estas son fijas

En caso de que no contemplemos las externalidades está claro que se cumplen tanto el supuesto (C) como el (P) en Bonnisseau y Meddeb (1999). El caso de la función de beneficios es similar al de dichos autores e incluso más abstracto ya que consideramos una función -compuesta- de tipo general. Lo mismo ocurre con los supuestos (BL), (WSA), (R) y (PR).

Por todas estas cuestiones observamos que si las externalidades fueren fijas o bien si estas no existiesen entonces nuestro modelo en este caso redundaría, sin más, en el modelo de Bonnisseau y Meddeb (1999), por lo que existiría un equilibrio tal como estos autores prueban. 


\section{3) Los conjuntos de producción son convexos}

En este caso, cualquiera sea $z_{-j} \in L_{\infty}(M, \mathrm{M}, \mu)^{m+n-1}$ tendremos que el conjunto $Y_{j}\left(z_{-j}\right)$ es convexo. A partir de allí la conducta de los productores queda definida por el principio maximizador $P M_{j}(z)=\left\{\pi \in S: \pi\left(y_{j}\right) \geq \pi(y) \forall y\right.$ $\left.\in Y_{j}\left(z_{-j}\right)\right\}$. En este caso observamos que se cumple el supuesto PR(i).

A continuación mostramos que $P M_{j}^{F}$ tiene un grafo cerrado. En efecto sean dos sucesiones, $\left(z^{n}\right)$ y $\left(p^{n}\right)$ en $Z^{F}$ y $S^{F}$ respectivamente, tal que para todo $n$ $\in \mathbb{N} p^{n} \in P M_{j}^{F}\left(z^{n}\right)$. Consideremos además que $z^{n} \rightarrow z$ y $p^{n} \rightarrow p$. De la definición de $P M_{j}^{F}$ tenemos que $p^{n}\left(y_{j}^{n}\right) \geq p^{n}\left(y_{j}\right) \forall y^{\prime} \in Y_{j}\left(z^{n}{ }_{-j}\right)$. Probemos que $p\left(y_{j}\right) \geq p(y)$ $\forall y \in Y_{j}^{F}\left(z_{-j}\right)$. En efecto sea $y \in Y_{j}^{F}\left(z_{-j}\right)$, como esta última es una correspondencia hemicontinua inferiormente en un espacio de dimensión finita entonces existe una sucesión $y^{n}$ convergente a $y$ tal que $y^{n} \in Y_{j}\left(z^{n}{ }_{-j}\right) \forall n \in \mathbb{N}$; sabemos que $p^{n}\left(y_{j}^{n}\right) \geq p^{n}\left(y^{n}\right)$ para cada $n \in \mathbb{N}$ de manera que tomando límites $p\left(y_{j}\right) \geq p(y)$. Como esto vale para cualquier $y \in Y_{j}^{F}\left(z_{-j}\right)$ entonces hemos probado que $p \in P M_{j}^{F}(z)$, luego $P M_{j}^{F}$ es cerrada.

A continuación veamos que se cumple el supuesto PR(iii). Comencemos con el punto a), de modo que dadas las condiciones explicitadas $\pi^{F(t)}\left(y_{j}^{F(t)}\right) \geq \pi^{F(t)}(y) \forall y \in Y_{j}\left(z^{F(t)}{ }_{-j}\right)$. Queremos probar que $\lim \pi^{F(t)}\left(y_{j}^{F(t)}\right) \geq \pi^{*}\left(y^{\prime}\right)$ $\forall y^{\prime} \in Y_{j}\left(z_{-j}^{*}\right)$. Supongamos que no, entonces existe algún $y^{\prime} \in Y_{j}\left(z_{-j}^{*}\right)$ tal que $\lim \pi^{F(t)}\left(y_{j}^{F(t)}\right)<\pi^{*}\left(y^{\prime}\right)$. En lo que sigue de esta demostración llamaremos $v_{j}$ a $\lim \pi^{F(t)}\left(y_{j}^{F(t)}\right)$. Si $v_{j}<\pi^{*}\left(y^{\prime}\right)$ entonces existe $\varepsilon>0$ tal que $v_{j}+\varepsilon<\pi^{*}\left(y^{\prime}\right)$ de donde se tiene que $v_{j}+\varepsilon / 2<\pi^{*}\left(y^{\prime}\right)-\varepsilon / 2$. Entonces como $\pi^{F(t)}\left(y_{j}^{F(t)}\right)$ tiende a $v_{j}$, existe un $t_{0} \in T$ tal que $\forall t \geq t_{0}, t \in T, \pi^{F(t)}\left(y_{j}^{F(t)}\right) \in B\left(v_{j} ; \varepsilon / 2\right)$ con $y_{j}^{F(t)} \in$ $\partial_{\infty} Y_{j}\left(z^{F(t)}{ }_{-j}\right)$. Sea $\varepsilon / 2$, a su turno como $\pi^{F(t)} \rightarrow \pi^{*}$ entonces existe $t_{1} \in T$ tal que $\forall t$ $\geq t_{1}, t \in T, \pi^{F(t)}\left(y^{\prime}\right) \in B\left(\pi^{*}\left(y^{\prime}\right) ; \varepsilon / 4\right)$ y por $\mathrm{P}\left(\right.$ ii) existe un $t_{2} \in T$ tal que $\forall t \geq t_{2}$, $Y_{j}\left(z^{F(t)}\right) \cap B\left(y^{\prime} ; \varepsilon / 4\right) \neq \varnothing^{22}$. Consideremos ahora $t_{3}>$ máx. $\left\{t_{0}, t_{1}, t_{2}\right\}$ en tal caso tenemos que para $y^{\prime \prime} \in Y_{j}\left(z^{F(t 3)}{ }_{-j}\right) \cap B\left(y^{\prime} ; \varepsilon / 4\right)$ y $\pi^{F(t 3)}\left(y^{\prime}\right) \in B\left(\pi^{*}\left(y^{\prime}\right) ; \varepsilon / 4\right)$ se

\footnotetext{
${ }^{22}$ Observemos que si en lugar de elegir el entorno $B\left(y^{\prime} ; \varepsilon / 4\right)$ que corresponde a la topología de la norma, hubiésemos escogido el entorno $U\left(y^{\prime}\right)=\left\{y \in L^{*}:\left|f(y)-f\left(y^{\prime}\right)<\varepsilon / 4\right| \forall i \in I\right\}$ correspondiente a la topología débil* habríamos arribado al mismo resultado ya que del anterior entorno se deduce que $y-\varepsilon / 4 \chi_{M}<y^{\prime}<y+\varepsilon / 4 \chi_{M}$.
} 
deduce que $\pi^{F(t 3)}\left(y^{\prime \prime}\right) \in B\left(\pi^{*}\left(y^{\prime}\right) ; \varepsilon / 2\right)$ y $\pi^{F(t 3)}\left(y_{j}^{F(t 3)}\right) \in B\left(v_{j} ; \varepsilon / 2\right)$. De todo ello tenemos las siguientes desigualdades

$$
\pi^{F(3)}\left(y_{j}^{F(t 3)}\right)<v_{j}+\varepsilon / 2<\pi^{*}\left(y^{\prime}\right)-\varepsilon / 2<\pi^{F(t 3)}\left(y^{\prime \prime}\right)
$$

Notemos que $y^{F(3)} \in \partial_{\infty} Y_{j}\left(z^{F(t 3)}{ }_{-j}\right)$ e $y^{\prime \prime} \in Y_{j}\left(z^{F(t 3)}{ }_{-j}\right)$ y como $\pi^{F(3)} \in$ $P M_{j}\left(z^{F(3)}\right)$ entonces $\pi^{F(t 3)}\left(y_{j}^{F(t 3)}\right) \geq \pi^{F(3)}\left(y^{\prime \prime}\right)$ lo cual es una contradicción con la desigualdad de arriba. Luego $\lim \pi^{F(t)}\left(y_{j}^{F(t)}\right) \geq \pi^{*}\left(y^{\prime}\right) \forall y^{\prime} \in Y_{j}\left(z_{-j}^{*}\right)$, de modo que, en particular, $\lim \pi^{F(t)}\left(y_{j}^{F(t)}\right) \geq \pi^{*}\left(y^{*}\right) \operatorname{con} y^{*} \in Y_{j}\left(z_{-j}^{*}\right)$.

Para probar que se cumple el supuesto PR(iii) (b) Supongamos que $\lim \pi^{F(t)}\left(y_{j}^{F(t)}\right)=\pi^{*}\left(y^{*}\right)$ entonces $\pi^{*}\left(y^{*}\right) \geq \pi^{*}\left(y^{\prime}\right) \forall y^{\prime} \in Y_{j}\left(z_{-j}^{*}\right)$ de modo que $\pi^{*} \in$ $P M_{j}\left(z^{*}\right) \subset$ S. Como $Y_{j}\left(z_{-j}^{*}\right)$ es convexo y dado el supuesto de libre disponibilidad $y^{*} \in \partial_{\infty} Y_{j}\left(z_{-j}^{*}\right)$.

\section{APENDICE C}

Rudimentos matemáticos utilizados en el trabajo:

(1) Una $\sigma$-álgebra $\mathrm{M}$ de subconjuntos de un conjunto $M$ es una clase de subconjuntos de $M$ que contienen tanto a $\varnothing$ como a $M$ y que es cerrado bajo las operaciones de complementación, unión numerable e intersección finita. Al par $(M, \mathrm{M})$ se lo llama espacio medible.

(2) Sea $(X, T)$ un espacio topológico. La $\sigma$-álgebra de Borel en $X$, denotada por $\mathrm{B}(X)$, es la $\sigma$-álgebra generada por los conjuntos T-abiertos de $X$.

(3) Dados dos espacios medibles $(M, \mathrm{M})$ y $\left(M^{\prime}, \mathrm{M}^{\prime}\right)$ una función $f: M \rightarrow M^{\prime}$ se dice medible si $f^{-1}(E)=\{\omega \in M: f(\omega) \in E\} \in \mathrm{M}$ para cada $E \in \mathrm{M}^{\prime}$.

(4) Sea $(M, M)$ un espacio medible. Una medida $\mu$ en $\mathrm{M}$ es una función de $\mathrm{M}$ en el intervalo $[0, \infty)$ tal que (i) $\mu(\varnothing)=0$ y (ii) $\mu\left(U_{i=1}^{\infty} E_{i}\right)=\sum_{i=1}^{\infty} \mu\left(E_{i}\right)$ donde $E_{i} \cap E_{i^{\prime}}=\varnothing$ para $i \neq i^{\prime}$. Al espacio $(M, \mathrm{M}, \mu)$ se lo llama espacio medida.

(5) $\operatorname{Si~} \mu(E)<\infty$ para todo $E \in \mathrm{M}$, entonces el espacio es $\sigma$-finito. 
(6) Llamamos a $L_{\infty}(M, \mathrm{M}, \mu)$ espacio de funciones en $M, \mathrm{M}$-medibles $\left[f^{-1}(E)=\right.$ $\{\omega \in M: f(\omega) \in E\} \in \mathrm{M}$ para cada $E \in \mathrm{B}(\mathbb{R})]$ y $\mu$-esencialmente acotadas $[\| f$ $\left.\|_{\infty}=\sup .\{\alpha \geq 0: \mu\{m \in M: f(m) \geq \alpha\}>0\}<\infty\right]$. Restringiremos el espacio al caso de las funciones de valor real.

(7) $\mathrm{Si} f$ y $g$ son dos funciones que pertenecen a $L_{\infty}(M, \mathrm{M}, \mu)$ tal que ambas son iguales en todo el dominio $M$ excepto en un conjunto de medida nula, entonces decimos que $f=g \mu$-a.e (traducido: casi en toda parte o c.t.p.) o bien que $f$ y $g$ son $\mu$-equivalentes. Con ello definimos a $\mathrm{L}_{\infty}(M, \mathrm{M}, \mu)$ como al espacio de clases de equivalencia (de $\mu$-equivalencia) de $\mathrm{L}_{\infty}(M, \mathrm{M}, \mu)$. Por otra parte $\mathrm{L}_{\infty}{ }^{+}(M, \mathrm{M}, \mu)=\left\{f \in L_{\infty}(M, \mathrm{M}, \mu): f(m) \geq 0 \mu\right.$-a.e $\}$. Todo lo anterior en este punto vale también para $\mathrm{L}_{1}(M, \mathrm{M}, \mu)$.

(8) El espacio $\mathrm{L}_{\infty}(M, \mathrm{M}, \mu)$ con la norma ||$.||_{\infty}$ es un espacio normado. Por su parte $\quad\left(\mathrm{L}_{\infty}(M, \mathrm{M}, \mu), \mathfrak{I}\right)$ es el espacio topológico donde $\mathfrak{I}$ es la topología inducida por la norma ||$\|_{\infty}$. Asimismo $\mathrm{L}_{\infty}(M, \mathrm{M}, \mu)$ es un espacio de Banach ya que en sí es un reticulado de Banach.

(9) Si $X$ es un espacio vectorial topológico, el dual topológico de $X, X^{*}$, es el conjunto de funcionales lineales continuos en $X$.

(10) Dado un par $(X, W)$ existe una topología, la menos fina, en $X$ tal que $W=$ $X^{*}$. A esta se la denota por $\sigma(X, W)$ y es la topología de la convergencia punto a punto en $W$, es decir, si $x^{v}$ es una red en $X$, entonces $x^{v}$ tiende a $x$ si y solo si $w . x^{v}$ tiende a $w \cdot x$ para todo $w$ en $W$. Llamamos a $\sigma(X, W)$ la topología débil en $X$, ya que es menos fina que la topología original. En adelante escribiremos directamente $\left(X, X^{*}\right)$ y $\sigma\left(X, X^{*}\right)$.

(11) Análogamente tendremos que $\sigma\left(X^{*}, X\right)$ es la topología débil en $X^{*}$, tal que si $p^{v}$ es una red en $X^{*}$, entonces $p^{v}$ tiende a $p$ si y solo si $p^{v}(x)$ tiende a $p(x)$ para todo $x$ en $X$. Se llama a $\sigma\left(X^{*}, X\right)$ topología débil* o topología débil estrella.

(12) Dado un par $(X, W)$ existe una topología, la mas fina, en $X$ tal que $W=X^{*}$. A esta se la denota por $\tau(X, W)$ y es definida como la topología de la convergencia uniforme, es decir, si $x^{v}$ es una red en $X$, entonces $x^{v}$ tiende a $x$ si y solo si $w$. $x^{v}$ tiende a $w \cdot x$ uniformemente para todo $w$ en cualquier subconjunto $\sigma(X, W)$-compacto de $W$. En adelante escribiremos directamente $\left(X, X^{*}\right)$ y $\tau\left(X, X^{*}\right)$. Similarmente $\tau\left(X^{*}, X\right)$ es la topología tal que si $p^{v}$ es una red en $X^{*}$, entonces $p^{v}$ tiende a $p$ si y solo si $p^{v}(x)$ tiende a $p(x)$ uniformemente para 
todo $x$ en un subconjunto $\sigma\left(X, X^{*}\right)$-compacto de $X$. Llamamos a $\tau$ topología Mackey.

(13) Sea $\mathrm{L}_{\infty}(M, \mathrm{M}, \mu)$, su dual topológico es $b a(M, \mathrm{M}, \mu)$, el espacio de las funciones de conjunto en $(M, \mathrm{M})$ acotadas, aditivas y absolutamente continuas. La topología $\sigma\left(\mathrm{L}_{\infty}, \mathrm{L}_{1}\right)$ es la topología débil* $\left(\mathrm{L}_{\infty}\right.$ dual de $\left.\mathrm{L}_{1}\right)$. Junto a la topología Mackey $\tau\left(\mathrm{L}_{\infty}, \mathrm{L}_{1}\right)$ son, respectivamente, la topología menos fina y la más fina para la cual el dual topológico de $\mathrm{L}_{\infty}(M, \mathrm{M}, \mu)$ es $\mathrm{L}_{1}(M, \mathrm{M}, \mu)$. Por su parte $\sigma\left(\mathrm{L}_{\infty}, b a\right)$ y $\sigma\left(b a, \mathrm{~L}_{\infty}\right)$ son las topologías débiles respectivas de $\mathrm{L}_{\infty}(M, \mathrm{M}$, н) у $b a(M, M, \mu)$.

(14) Sea $(X, T)$ un espacio topológico, si $x^{v}$ es una red en $X T$-convergente a $x$ lo escribiremos $x^{v T} \rightarrow x$. Los casos $x^{v}{ }^{w} \rightarrow x$ y $x^{v} w^{*} \rightarrow x$ son los respectivos a $(X$, $\left.\sigma\left(X, X^{*}\right)\right)$ y $\left(X^{*}, \sigma\left(X^{*}, X\right)\right)$

(15) Sea $A$ un conjunto, definiremos al conjunto de partes de $A$ como $2^{A}$. Sea $(X, \mathrm{~T})$ un espacio topológico y sea $A \subset X$, entonces $\operatorname{cl} A$ denota la $\mathrm{T}$-clausura de $A$, intA el $\mathrm{T}$-interior de $A$ y $\partial A$ la $\mathrm{T}$-frontera de $A$. Si $X$ es de dimensión infinita escribimos la frontera como $\partial_{\infty}$.

(16) Sea $x \in X,(X, \mathrm{~T})$ espacio topológico. Entonces $U$ es un $\mathrm{T}$-entorno de $x$ si $x \in \operatorname{int} U$.

(17) Sea la correspondencia $\mathrm{C}:(X, \mathrm{~T}) \rightarrow 2^{X^{\prime}}$, donde $(X, \mathrm{~T})$ y $\left(X^{\prime}, \mathrm{T}{ }^{\prime}\right)$ son espacios topológicos, entonces $\mathrm{C}$ es $\mathrm{T}$ - hemicontinua superior en $x \in X$, si $\mathrm{C}(x)$ $\neq \varnothing$ y para todo $\mathrm{T}^{\prime}$-entorno $U$ de $\mathrm{C}(x)$, existe un $\mathrm{T}$-entorno $V$ de $x$, tal que $\mathrm{C}(z)$ $\subset U$ para todo $z$ en $V$. La correspondencia es $\mathrm{T}$ - hemicontinua superior si es $\mathrm{T}$ - hemicontinua superior en todo $x \in X$.

(18) Sea la correspondencia $\mathrm{C}:(X, \mathrm{~T}) \rightarrow 2^{X^{\prime}}$, donde $(X, \mathrm{~T})$ y $\left(X^{\prime}, \mathrm{T}{ }^{\prime}\right)$ son espacios topológicos, entonces $\mathrm{C}$ es $\mathrm{T}$ - hemicontinua inferior en $x \in X$, si $\mathrm{C}(x)$ $\neq \varnothing$ y para todo conjunto $\mathrm{T}$ '-abierto $G$ tal que $\mathrm{C}(x) \cap G \neq \varnothing$, existe un $\mathrm{T}$ entorno $V$ de $x$, tal que $\mathrm{C}(z) \cap G \neq \varnothing$ para todo $z$ en $V$. La correspondencia es $\mathrm{T}$ - hemicontinua inferior si es T - hemicontinua inferior en todo $x \in X$.

(19) Sea la correspondencia $\mathrm{C}:(X, \mathrm{~T}) \rightarrow 2^{X^{\prime}}$, donde $(X, \mathrm{~T})$ y $\left(X^{\prime}, \mathrm{T}{ }^{\prime}\right)$ son espacios topológicos, entonces $\mathrm{C}$ es $\mathrm{T}$ - cerrada si $x^{v}$ y $x^{\prime v}$ son redes en $X$ y $X^{\prime}$ respectivamente con $x^{v \mathrm{~T}} \rightarrow x$ en $X$ y $x^{\prime v}{ }^{\mathrm{T}}{ }^{\prime} \rightarrow x^{\prime}$ en $X^{\prime}, \mathrm{y} x^{\prime v} \in \mathrm{C}\left(x^{v}\right)$ para todo $v$, entonces $x^{\prime} \in \mathrm{C}(x)$. 


\section{REFERENCIAS BIBLIOGRAFICAS}

Ash, R., (1972), Real Analysis and Probability, San Diego, California, Academic Press, Inc.

Bewley, T., (1972), "Existence of Equilibria in Economies with Infinitely Many Commodities", Journal of Economic Theory, 4, pp. 514-540.

Bonnisseau, J., M., (1997), "Existence of Equilibria in Economies with Externalities and Nonconvexities", Set Valued Analysis 5, pp. 209226.

Bonnisseau, J., M., (2000), "The Marginal Pricing Rule in Economies with Infinitely Many Commodities", Working paper, CERMSEM, Université Paris I, pp.1-20.

Bonnisseau, J., M., y Cornet, B., (1988), "Existence of Equilibrium when Firms Follow Bounded Losses Pricing Rules", Journal of Mathematical Economics, 17, pp. 119-147.

Bonnisseau, J., M., y Cornet, B., (1990), "Existence of Marginal Cost Pricing Equilibria: The Nonsmooth Case", International Economic Review, Vol. 31, (3), pp. 685-708.

Bonnisseau, J., M., y Meddeb, M., (1999), "Existence of Equilibria in Economies with Increasing Returns and Infinitely Many Commodities", Journal of Mathematical Economics, Vol. 31, pp. 287 307.

Bonnisseau, J., M., y Médecin, M., (2001), "Existence of marginal pricing equilibria in economies with externalities and non-convexities", Journal of Mathematical Economics, Vol. 36, pp.271-294.

Clarke, F., (1983), Optimization and Nonsmooth Analysis, Canadian Mathematical Society, Series of Monographs and Advanced Texts.

Cornet, B., (1988), "General Equilibrium Theory and Increasing Returns: Presentation", Journal of Mathematical Economics, Vol. 17, pp.103118.

Debreu, G., (1959), Theory of Value, New Haven: Yale University Press.

Dunford, N., and Schwarz, J., (1958), Linear Operators, Part I, WileyInterscience, New York.

Hildenbrand, W., (1974), Core and Equilibria of a Large Economy, Princeton University Press, Princeton, New Jersey.

Joseph, J., (1978), "Multifunctions and Graphs", Pacific Journal of Mathematics, Vol. 79, (2).

Kamiya, K., (1988), "On The Survival Assumption in Marginal (Cost) Pricing”, Journal of Mathematical Economics, Vol.17, pp.261-273. 
Kelley, J., (1962), Topología General, Editorial Universitaria de Buenos Aires, Argentina. Traducción de General Topology, (1955), D. Van Nostrand, Princeton, New Jersey.

Kolmogorov, A. y Fomin, S., (1957), Elements of the Theory of Functions and Functional Analysis, Volume 1 Metric and Normed Spaces. Graylock Press, Rochester, N. Y. Traducido de la primera version rusa (1954) Èlementy Teorii Funkciŭ i Funkcionalnogo Analiza., I. Metričeskie i Normirovannye Prostranstva.

Mas-Colell, A., y Zame, W., (1991), "Equilibrium Theory in Infinite Dimensional Spaces", Hildenbrand, W., and Sonnenschein, H., Handbook of Mathematical Economics, Vol. IV,, 1836-1898, Amsterdam, North-Holland.

Mas-Colell, A., Whinston, M. y Green, J., (1995), Microeconomic Theory, Oxford University Press.

Noguchi, M., (1998), "Economies with a continuum of agents with the commodity-price pairing $\left(l_{\infty}, l_{1}\right)$ ", Journal of Mathematical Economics, Vol. 28, pp. 265-287.

Nowak, M., (1989) "On the Finest Lebesgue Topology on the Space of essentially bounded measurable functions". Pacific Journal of Mathematics, Vol. 140, (1) pp. 155-161.

Schaefer, H., (1971), Topological Vector Spaces, New York and Berlin, Springer-Verlag.

Vulikh, B. (1963), Introduction to Functional Analysis for Scientists and Technologists, Addison-Wesley, London, Traducción al inglés de Vvedeniye v funktisional'nyi analiz, (1958), Moscow, Fizmatgiz. 